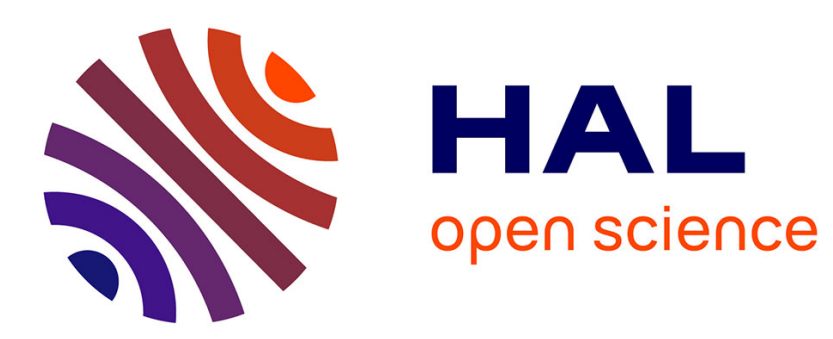

\title{
Instability of an inverse problem for the stationary Navier-Stokes equations
}

Fabien Caubet

\section{To cite this version:}

Fabien Caubet. Instability of an inverse problem for the stationary Navier-Stokes equations. SIAM Journal on Control and Optimization, 2013, 51 (4), pp.2949-2975. hal-00696174

\section{HAL Id: hal-00696174 \\ https://hal.science/hal-00696174}

Submitted on 11 May 2012

HAL is a multi-disciplinary open access archive for the deposit and dissemination of scientific research documents, whether they are published or not. The documents may come from teaching and research institutions in France or abroad, or from public or private research centers.
L'archive ouverte pluridisciplinaire HAL, est destinée au dépôt et à la diffusion de documents scientifiques de niveau recherche, publiés ou non, émanant des établissements d'enseignement et de recherche français ou étrangers, des laboratoires publics ou privés. 


\title{
Instability of an inverse problem for the stationary Navier-Stokes equations
}

\author{
Fabien Caubet*
}

April 3, 2012

\begin{abstract}
This paper provides a theoretical study of the detection of an object immersed in a fluid when the fluid motion is governed by the stationary Navier-Stokes equations with non homogeneous Dirichlet boundary conditions. To solve this inverse problem, we make a boundary measurement on a part of the exterior boundary. First, we present an identifiability result. We then use a shape optimization method: in order to identify the obstacle, we minimize a nonlinear least squares criterion. Thus, we prove the existence of the first order shape derivative of the state, we characterize it and deduce the gradient of the least squares functional. Finally, we study the stability of this setting doing a shape sensitivity analysis of order two. Hence, we prove the existence of the second order shape derivatives and we give the expression of the shape Hessian at possible solutions of the original inverse problem. Then, the compactness of the Riesz operator corresponding to this shape Hessian is shown and the ill-posedness of the identification problem follows. This explains the need of regularization to solve numerically this problem.
\end{abstract}

Keywords: stationary Navier-Stokes equations, order two shape sensitivity, geometric inverse problem, sensitivity with respect to the domain, shape calculus.

AMS Classification: 35R30, 49Q10, 49Q12, 35Q30, (76D05).

\section{Introduction and notations}

Motivations. Inverse problems are a source of many investigations, from both a mathematical and industrial perspective. One of the topics studied in this domain is the detection of an obstacle immersed in a fluid. A concrete application is the detection of shoals of fish, gas bubbles in a fluid (during mould fillings for example) or water bubbles in oil in a pipe for example.

A method to solve these inverse problems, particularly in the case of fluid mechanics, is the shape optimization method. The first step to solve such a problem is to prove the existence of the shape derivatives. In 1991, Simon does it rigorously in the case of a Stokes flow in [24 and the Navier-Stokes case is treated by Bello et al. in 1992 in [7] and [8]. Then, in 2005, Alvarez et al. study in [3] the following inverse problem: an inaccessible rigid body $\omega$ is immersed in a viscous fluid, in such a way that $\omega$ plays the role of an obstacle around which the fluid is flowing in a greater bounded domain $\Omega$. They wish to determine $\omega$ (i.e. its shape and location) via boundary measurement on the boundary $\partial \Omega$. Under reasonable smoothness assumptions on $\Omega$ and $\omega$, they prove that one can identify $\omega$

*LMAP, UMR 5142, Université de Pau et des Pays de l'Adour, CNRS, F-64013 Pau, France 
via the measurement of both the velocity of the fluid and the Cauchy forces on some part of the boundary $\partial \Omega$. They also give a directional stability result for the inverse problem (see [3, Theorem 1.3]). The calculus of shape derivative concerning the steady-state NavierStokes equations is treated for an optimal shape problem in 2008 by Henrot et al. in [20] and [21]. In 2008, Conca et al. investigate in [10] the problem of the detection of a moving obstacle in a perfect fluid by a boundary measurement. They show that, when the obstacle is a ball, one may identify the position and the velocity of its center of mass from a single boundary measurement. In 2010, using complex analysis, Conca et al. prove in [11 that this result can not be generalized to any solid. However, they extend it to moving ellipses and they prove that when the solid enjoys some symmetry properties, it can be partially detected. In 2011, Badra et al. prove in [6] the instability of the detection of an object immersed in a fluid in the Stokes case with Dirichlet or Neumann boundary conditions.

Numerical resolution of inverse problems often leads to difficulties. This paper explains why one has to expect numerical difficulties to detect and reconstruct an obstacle immersed in a fluid when the fluid motion is governed by the stationary Navier-Stokes equations. Indeed, we focus on the question of stability of a shape optimization problem used to solve this inverse problem. The aim of this paper is to adapt the results presented in the Stokes case by Badra et al. in [6] to the nonlinear Navier-Stokes case. We here deal with non homogeneous Dirichlet boundary conditions. The method that we adopt to solve this inverse problem is to make a boundary measurement on a part of the exterior boundary. Following previous works on electrical impedance tomography (by Eppler et al. in [14] and Afraites et al. in [1, [2]) or on the same topic in the Stokes case (by Badra et al. in [6]), our strategy is then to minimize a least squares functional. We then prove the existence of the first and second order shape derivatives and characterize the gradient of this functional. Here, we give a general mathematical framework to prove the existence of the shape derivatives in this kind of problem using weighted spaces. It permits to prove an intuitive idea: the regularity of the exterior boundary does not have importance. In order to study the stability, we compute the shape Hessian at a critical point. We then prove that the Riesz operator corresponding to this shape Hessian is compact, which means that the functional is degenerate for the high frequencies.

This paper is organized as follows. We first introduce the general notation that we adopt. In Section 2 we recall some preliminary results whose the arguments used in this work strongly depend: existence and uniqueness theorems and local regularity results. The problem setting is exposed in Section 3 where we introduce the needed functional tools. Section 4 is devoted to the identifiability result. The discussion of the first order shape derivatives is made in Section 5. We prove the existence of the first order shape derivatives, we characterize them and, introducing an adjoint problem, we compute the gradient of the least squares functional. Moreover, we prove the existence of the local first variation of the solution of the adjoint problem which is a linearized Navier-Stokes equations. The material related to the higher order shape derivatives is combined in Section 6. We present the existence of higher order shape derivatives and characterize the shape Hessian at possible solutions of the original inverse problem. The last section 7 justfies the ill-posedness of the problem. We state that the shape Hessien has a pathological behavior: the Riesz operator corresponding to this shape Hessian is compact.

General notation. Let us introduce the notations that we adopt in this paper. We denote by $\mathrm{L}^{p}, \mathrm{~W}^{m, p}$ and $\mathrm{H}^{s}$ the usual Lebesgue and Sobolev spaces. For $k \in \mathbb{N}$ and an open set $\Omega \subset \mathbb{R}^{N}(N=2$ or 3$)$, the space $C_{c}^{k}(\Omega)$ is defined as the set of compactly supported functions in $\Omega$ having continuous derivatives up to order $k$ and $\|\cdot\|_{k, \infty}$ is its natural 
norm. For $u \in C_{c}^{k}(\Omega), \operatorname{supp}(u)$ denotes the support of $u$. We also denote by $\mathcal{D}(\Omega)$ the space of $C^{\infty}$ functions compactly supported in $\Omega$. We note in bold the vectorial functions and spaces: $\mathbf{L}^{p}, \mathbf{W}^{m, p}, \mathbf{H}^{s}$, etc. Moreover we note respectively $\langle\cdot, \cdot\rangle_{\Omega}$ and $\langle\cdot, \cdot\rangle_{\partial \Omega}$ the duality product between $\mathbf{H}^{-1}(\Omega)$ and $\mathbf{H}_{0}^{1}(\Omega)$ and the duality product between $\mathbf{H}^{-1 / 2}(\partial \Omega)$ and $\mathbf{H}^{1 / 2}(\partial \Omega)$. We denote by $|\Omega|$ the measure of $\Omega$. Moreover, $\mathbf{n}$ represents the external unit normal to $\partial \Omega$, and for a smooth enough function $u$, we note $\partial_{\mathbf{n}} u$ the normal derivative of $u$. We also denote by $\mathcal{M}_{N, N}$ the space of matrices of size $N \times N$. Moreover, $b_{\Omega}(\cdot, \cdot, \cdot)$ is the trilinear continuous form on $\mathbf{H}^{1}(\Omega) \times \mathbf{H}^{1}(\Omega) \times \mathbf{H}^{1}(\Omega)$ defined by

$$
b_{\Omega}(\boldsymbol{u}, \boldsymbol{v}, \boldsymbol{w})=\sum_{i, j=1}^{N} \int_{\Omega} \boldsymbol{u}_{i} \frac{\partial \boldsymbol{v}_{j}}{\partial x_{i}} \boldsymbol{w}_{j} .
$$

The subscript of $b$ is used to indicate the domain of integration if there are ambiguities. Finally, for $k \geq-1$ and $m \geq 0$ integers with $k<m$ and for two open subsets $\Omega_{1}$ and $\Omega_{2}$ such that $\Omega_{2} \subset \Omega_{1}$, we note $\mathrm{X}^{k, m}\left(\Omega_{1}, \Omega_{2}\right)$ the space of functions in $\mathrm{H}^{k}\left(\Omega_{1}\right)$ such that their restriction to $\Omega_{2}$ belongs to $\mathrm{H}^{m}\left(\Omega_{2}\right)$. This space endowed with the norm

$$
\|u\|_{\mathrm{X}^{k, m}\left(\Omega_{1}, \Omega_{2}\right)}:=\left(\|u\|_{\mathrm{H}^{k}\left(\Omega_{1}\right)}^{2}+\|u\|_{\mathrm{H}^{m}\left(\Omega_{2}\right)}^{2}\right)^{1 / 2},
$$

is hilbertian.

\section{Remainder of some preliminary useful results}

\subsection{Some results on the Navier-Stokes problem}

For $\Omega$ an open subset of $\mathbb{R}^{N}(N=2,3)$, we define

$$
\mathrm{L}_{0}^{2}(\Omega):=\left\{q \in \mathrm{L}^{2}(\Omega) ; \int_{\Omega} q=0\right\} .
$$

We first recall a classical existence theorem which can be found in [17, Theorem IV.2.3]. We also refer to [25], 16] or [12] for classical results concerning the Navier-Stokes equations and to [5] for the $\mathrm{L}^{p}$ theory.

Theorem 2.1 (Existence of a solution. Girault, Raviart, [17]). Let $N=2,3, \Omega$ be a bounded connected Lipschitz open set of $\mathbb{R}^{N}, \Gamma_{i}, i=1, \ldots, I$, the connected components of $\partial \Omega$ and $\nu>0$. Let $\boldsymbol{f} \in \mathbf{H}^{-1}(\Omega)$ and $\boldsymbol{g} \in \mathbf{H}^{1 / 2}(\partial \Omega)$ satisfy the condition

$$
\int_{\Gamma_{i}} \boldsymbol{g} \cdot \mathbf{n}=0 \quad \forall i=1, \ldots, I .
$$

Then, the Navier-Stokes problem

$$
\left\{\begin{array}{rlll}
-\nu \Delta \boldsymbol{u}+(\boldsymbol{u} \cdot \nabla) \boldsymbol{u}+\nabla p & = & \text { in } \Omega \\
\operatorname{div} \boldsymbol{u} & = & & \text { in } \Omega \\
\boldsymbol{u} & =\boldsymbol{g} & \text { on } \partial \Omega
\end{array}\right.
$$

admits at least a solution $(\boldsymbol{u}, p) \in \mathbf{H}^{1}(\Omega) \times \mathrm{L}_{0}^{2}(\Omega)$.

In order to prove a regularity result, we first recall a classical corollary of the Sobolev imbeddings and we deduce a practical result. 
Theorem 2.2. Assume that $\Omega$ is a bounded Lipschitz open subset of $\mathbb{R}^{N}$. Let $s_{1}, s_{2} \in \mathbb{R}^{+}$ and $s \in\left[-1,+\infty\left[\right.\right.$ such that $s_{1} \geq s, s_{2} \geq s$ and either

$$
s_{1}+s_{2}-s \geq \frac{N}{2}, \quad s_{i}-s>0 \quad i=1,2
$$

or

$$
s_{1}+s_{2}-s>\frac{N}{2}, \quad s_{i}-s \geq 0 \quad i=1,2 .
$$

Then $(u, v) \mapsto u \cdot v$ is a continuous bilinear map from $\mathrm{H}^{s_{1}}(\Omega) \times \mathrm{H}^{s_{2}}(\Omega)$ into $\mathrm{H}^{s}(\Omega)$.

Proof. The usual result is given for $m_{1}=s_{1}, m_{2}=s_{2}$ and $m=s+1$ integers (see [17, Corollary I.1.1]) but we deduce this result with an interpolation argument (see for example [9. Theorem 6.4.5]).

It leads to the following result:

Corollary 2.3. Let $\Omega$ be a bounded Lipschitz open subset of $\mathbb{R}^{N}(N=1,2,3)$ and $s \in \mathbb{R}^{+}$. The mapping

$$
(u, v) \in \mathrm{H}^{1+s}(\Omega) \times \mathrm{H}^{s}(\Omega) \mapsto u \cdot v \in \mathrm{H}^{s-1 / 2}(\Omega)
$$

is bilinear continuous. In particular, the mapping

$$
\boldsymbol{u} \in \mathbf{H}^{1+s} \mapsto(\boldsymbol{u} \cdot \nabla) \boldsymbol{u} \in \mathbf{H}^{s-1 / 2}(\Omega)
$$

is continuous.

Corollary 2.4 (Regularity of the solution). Let $\Omega$ be a bounded connected open set of $\mathbb{R}^{N}$ $(N=2,3)$ with a $C^{k, 1}$ boundary $(k \in \mathbb{N})$ and $\nu>0$. Let $\boldsymbol{f} \in \mathbf{H}^{k-1}(\Omega)$ and $\boldsymbol{g} \in \mathbf{H}^{k+1 / 2}(\partial \Omega)$ satisfying the condition (2.1). Then Problem (2.2) admits a solution $(\boldsymbol{u}, p)$ which belongs in $\mathbf{H}^{k+1}(\Omega) \times\left(\mathrm{H}^{k}(\Omega) \cap \mathrm{L}_{0}^{2}(\Omega)\right)$.

Proof. We will use the regularity of the solutions of the Stokes equations and proceed by induction. We know using Theorem 2.1 that the result is true for $k=0$.

Let us assume that the result is true for some $k \in \mathbb{N}$. Then $\boldsymbol{u} \in \mathbf{H}^{k+1}(\Omega)$ and we have

$$
\left\{\begin{aligned}
-\nu \Delta \boldsymbol{u}+\nabla p & =\boldsymbol{f}-(\boldsymbol{u} \cdot \nabla) \boldsymbol{u} & & \text { in } \Omega \\
\operatorname{div} \boldsymbol{u} & =0 & & \text { in } \Omega \\
\boldsymbol{u} & =\boldsymbol{g} & & \text { on } \partial \Omega .
\end{aligned}\right.
$$

Let us prove that $(\boldsymbol{u} \cdot \nabla) \boldsymbol{u} \in \mathbf{H}^{k}(\Omega)$. Since $\boldsymbol{u} \in \mathbf{H}^{k+1}(\Omega)$, then, according to Corollary 2.3 . $(\boldsymbol{u} \cdot \nabla) \boldsymbol{u} \in \mathbf{H}^{k-1 / 2}(\Omega)$. Therefore, using the regularity of the solutions of the Stokes equations (see for example [4, Theorem 4.8]), $\boldsymbol{u} \in \mathbf{H}^{k+3 / 2}(\Omega)$. The same argument gives $(\boldsymbol{u} \cdot \nabla) \boldsymbol{u} \in \mathbf{H}^{k}(\Omega)$ and $(\boldsymbol{u}, p) \in \mathbf{H}^{k+2}(\Omega) \times \mathrm{H}^{k+1}(\Omega)$ which concludes the proof.

Let us recall a uniqueness result (see [16, Theorem VIII.2.1]):

Theorem 2.5 (Unicity of the solution. Galdi, [16]). Let us consider Problem (2.2). If $\nu>c_{b}(\Omega)\|\nabla \boldsymbol{u}\|_{\mathbf{L}^{2}(\Omega)}$ where

$$
c_{b}(\Omega):=\left\{\begin{array}{ccc}
\frac{|\Omega|^{1 / 2}}{2} & \text { if } & N=2 \\
\frac{2 \sqrt{2}|\Omega|^{1 / 6}}{3} & \text { if } & N=3,
\end{array}\right.
$$

then the solution given by Corollary 2.4 is unique. 
Let us prove the following local regularity result:

Theorem 2.6 (Local regularity for the Navier-Stokes equations). Let $k \in \mathbb{N}^{*}, \nu>0, \Omega$ be a bounded connected Lipschitz open set of $\mathbb{R}^{N}(N=2,3)$ and $\omega \subset \subset \Omega$ be an open set with a $C^{k, 1}$ boundary such that $\Omega \backslash \bar{\omega}$ is connected. Let $\mathcal{C}$ and $\mathcal{C}^{\prime}$ be two smooth open subsets of $\Omega \backslash \bar{\omega}$ such that $\partial \omega \subset \partial \mathcal{C}, \partial \omega \subset \partial \mathcal{C}^{\prime}, \overline{\mathcal{C}} \backslash \partial \omega \subset \mathcal{C}^{\prime}$ and $\overline{\mathcal{C}^{\prime}} \subset \Omega$. Let

$$
\left(\boldsymbol{f}, \boldsymbol{h}_{\text {ext }}, \boldsymbol{h}_{\text {int }}\right) \in \mathbf{X}^{-1, k-1}\left(\Omega \backslash \bar{\omega}, \mathcal{C}^{\prime}\right) \times \mathbf{H}^{1 / 2}(\partial \Omega) \times \mathbf{H}^{k+1 / 2}(\partial \omega),
$$

such that

$$
\int_{\partial \Omega} \boldsymbol{h}_{\boldsymbol{e x t}} \cdot \mathbf{n}+\int_{\partial \omega} \boldsymbol{h}_{\boldsymbol{i n t}} \cdot \mathbf{n}=0 .
$$

Then every solution $(\boldsymbol{u}, p) \in \mathbf{H}^{1}(\Omega \backslash \bar{\omega}) \times \mathrm{L}_{0}^{2}(\Omega \backslash \bar{\omega})$ of the Navier-Stokes problem

$$
\left\{\begin{aligned}
-\nu \Delta \boldsymbol{u}+(\boldsymbol{u} \cdot \nabla) \boldsymbol{u}+\nabla p & =\boldsymbol{f} & & \text { in } \Omega \backslash \bar{\omega} \\
\operatorname{div} \boldsymbol{u} & =0 & & \text { in } \Omega \backslash \bar{\omega} \\
\boldsymbol{u} & =\boldsymbol{h}_{\text {ext }} & & \text { on } \partial \Omega \\
\boldsymbol{u} & =\boldsymbol{h}_{\text {int }} & & \text { on } \partial \omega
\end{aligned}\right.
$$

belongs to $\mathbf{H}^{k+1}(\mathcal{C}) \times \mathrm{H}^{k}(\mathcal{C})$.

Proof. We will use the local regularity of the solutions of the Stokes equations (see for example [16, Theorem IV.5.1]).

First let us consider the case $k=1$. We then proceed by induction for $k>1$. We have

$$
\left\{\begin{aligned}
-\nu \Delta \boldsymbol{u}+\nabla p & =\boldsymbol{f}-(\boldsymbol{u} \cdot \nabla) \boldsymbol{u} & & \text { in } \Omega \\
\operatorname{div} \boldsymbol{u} & =0 & & \text { in } \Omega \\
\boldsymbol{u} & =\boldsymbol{g} & & \text { on } \partial \Omega .
\end{aligned}\right.
$$

Let us prove that $(\boldsymbol{u} \cdot \nabla) \boldsymbol{u} \in \mathbf{X}^{-1,0}\left(\Omega \backslash \bar{\omega}, \mathcal{C}^{\prime}\right)$. Since $\boldsymbol{u} \in \mathbf{H}^{1}(\Omega \backslash \bar{\omega})$, then, according to Corollary 2.3, $(\boldsymbol{u} \cdot \nabla) \boldsymbol{u} \in \mathbf{H}^{-1 / 2}(\Omega \backslash \bar{\omega})$. Therefore, using the local regularity of the solutions of the Stokes equations, $\boldsymbol{u} \in \mathbf{X}^{1,3 / 2}(\Omega \backslash \bar{\omega}, \mathcal{V})$, where $\mathcal{V}$ is a smooth open subset of $\Omega \backslash \bar{\omega}$ such that $\mathcal{C} \subset \subset \mathcal{V} \subset \subset \mathcal{C}^{\prime}$. Therefore $\boldsymbol{u} \in \mathbf{X}^{1,3 / 2}(\Omega \backslash \bar{\omega}, \mathcal{V})$. The same argument gives $(\boldsymbol{u} \cdot \nabla) \boldsymbol{u} \in \mathbf{X}^{-1 / 2,0}(\Omega \backslash \bar{\omega}, \mathcal{V})$ and $(\boldsymbol{u}, p) \in \mathbf{X}^{1,2}(\Omega \backslash \bar{\omega}, \mathcal{C}) \times \mathbf{X}^{0,1}(\Omega \backslash \bar{\omega}, \mathcal{C})$.

\subsection{Some results on the linearized Navier-Stokes problem}

Theorem 2.7 (Existence and uniqueness of the solution of the linearized Navier-Stokes equations). Let $\Omega$ be a bounded connected Lipschitz open set of $\mathbb{R}^{N}(N=2,3)$. Let us consider $\boldsymbol{f} \in \mathbf{H}^{-1}(\Omega), g \in \mathrm{L}^{2}(\Omega)$ and $\boldsymbol{h} \in \mathbf{H}^{1 / 2}(\partial \Omega)$ such that

$$
\int_{\Omega} g=\int_{\partial \Omega} \boldsymbol{h} \cdot \mathbf{n} \text {. }
$$

Let $\boldsymbol{v} \in \mathbf{H}^{1}(\Omega)$ such that $\nu>c_{b}(\Omega)\|\boldsymbol{v}\|_{\mathbf{H}^{1}(\Omega)}$, where $c_{b}(\Omega)$ is defined by $(2.3)$. Then the problem

$$
\left\{\begin{array}{rlll}
-\nu \Delta \boldsymbol{u}+(\boldsymbol{u} \cdot \nabla) \boldsymbol{v}+(\boldsymbol{v} \cdot \nabla) \boldsymbol{u}+\nabla p & =\boldsymbol{f} & \text { in } \Omega \\
\operatorname{div} \boldsymbol{u} & =g & \text { in } \Omega \\
\boldsymbol{u} & =\boldsymbol{h} & \text { on } \partial \Omega
\end{array}\right.
$$

admits a unique solution $(\boldsymbol{u}, p) \in \mathbf{H}^{1}(\Omega) \times \mathrm{L}_{0}^{2}(\Omega)$ and there exists a constant $C$ (which depends on $N, \Omega, \nu$, and $\boldsymbol{v})$ such that

$$
\|\boldsymbol{u}\|_{\mathbf{H}^{1}(\Omega)}+\|p\|_{\mathrm{L}^{2}(\Omega)} \leq C\left(\|\boldsymbol{f}\|_{\mathbf{H}^{-1}(\Omega)}+\|g\|_{\mathrm{L}^{2}(\Omega)}+\|\boldsymbol{h}\|_{\mathbf{H}^{1 / 2}(\partial \Omega)}\right) .
$$


Proof. Using a lifting argument (see [4, Lemma 3.3]), existence and uniqueness of the solution of problem (2.4) reduce to existence and uniqueness of the solution of the homogeneous problem. We then use Lax-Milgram's Theorem and finally De Rham's Theorem (see for example [4, Lemma 2.7]) to obtain the corresponding pressure $p$.

Theorem 2.8 (Local regularity for the linearized Navier-Stokes equations). Let $k \in \mathbb{N}^{*}$, $\nu>0, \Omega$ be a bounded connected Lipschitz open set of $\mathbb{R}^{N}(N=2,3)$ and $\omega \subset \subset \Omega$ be an open set with a $C^{k, 1}$ boundary such that $\Omega \backslash \bar{\omega}$ is connected. Let $\mathcal{C}$ and $\mathcal{C}^{\prime}$ be two smooth open subsets of $\Omega \backslash \bar{\omega}$ such that $\partial \omega \subset \partial \mathcal{C}, \partial \omega \subset \partial \mathcal{C}^{\prime}, \overline{\mathcal{C}} \backslash \partial \omega \subset \mathcal{C}^{\prime}$ and $\overline{\mathcal{C}^{\prime}} \subset \Omega$. Let

$$
\left(\boldsymbol{f}, \boldsymbol{g}, \boldsymbol{h}_{\text {ext }}, \boldsymbol{h}_{\boldsymbol{i n t}}\right) \in \mathbf{X}^{-1, k-1}\left(\Omega \backslash \bar{\omega}, \mathcal{C}^{\prime}\right) \times \mathrm{X}^{0, k}\left(\Omega \backslash \bar{\omega}, \mathcal{C}^{\prime}\right) \times \mathbf{H}^{1 / 2}(\partial \Omega) \times \mathbf{H}^{k+1 / 2}(\partial \omega),
$$

such that

$$
\int_{\Omega \backslash \bar{\omega}} g=\int_{\partial \Omega} \boldsymbol{h}_{\text {ext }} \cdot \mathbf{n}+\int_{\partial \omega} \boldsymbol{h}_{\boldsymbol{i n t}} \cdot \mathbf{n} .
$$

Let $\boldsymbol{v} \in \mathbf{H}^{1}(\Omega \backslash \bar{\omega})$ such that $\boldsymbol{v}_{\mid \mathcal{C}^{\prime}} \in \mathbf{H}^{k+1}\left(\mathcal{C}^{\prime}\right)$. Every solution $(\boldsymbol{u}, p) \in \mathbf{H}^{1}(\Omega \backslash \bar{\omega}) \times \mathrm{L}_{0}^{2}(\Omega \backslash \bar{\omega})$ of the linearized Navier-Stokes problem

$$
\left\{\begin{aligned}
-\nu \Delta \boldsymbol{u}+(\boldsymbol{v} \cdot \nabla) \boldsymbol{u}+(\boldsymbol{u} \cdot \nabla) \boldsymbol{v}+\nabla p & =\boldsymbol{f} & & \text { in } \Omega \backslash \bar{\omega} \\
\operatorname{div} \boldsymbol{u} & =g & & \text { in } \Omega \backslash \bar{\omega} \\
\boldsymbol{u} & =\boldsymbol{h}_{\text {ext }} & & \text { on } \partial \Omega \\
\boldsymbol{u} & =\boldsymbol{h}_{\text {int }} & & \text { on } \partial \omega
\end{aligned}\right.
$$

belongs to $\mathbf{H}^{k+1}(\mathcal{C}) \times \mathrm{H}^{k}(\mathcal{C})$. Moreover, if $\nu>c_{b}(\Omega \backslash \bar{\omega})\|\boldsymbol{v}\|_{\mathbf{H}^{1}(\Omega \backslash \bar{\omega})}$ (where $c_{b}(\Omega \backslash \bar{\omega})$ is defined analogously to (2.3)), then the solution is unique and there exists a constant $C$ (which depends on $N, \Omega, \nu$, and $\boldsymbol{v}$ ) such that

$$
\begin{aligned}
\|\boldsymbol{u}\|_{\mathbf{H}^{k+1}(\mathcal{C})}+\|p\|_{\mathrm{H}^{k}(\mathcal{C})} \leq C\left(\|\boldsymbol{f}\|_{\mathbf{X}^{-1, k-1}\left(\Omega \backslash \bar{\omega}, \mathcal{C}^{\prime}\right)}\right. & +\|g\|_{\mathrm{X}^{0, k}\left(\Omega \backslash \bar{\omega}, \mathcal{C}^{\prime}\right)} \\
& \left.+\left\|\boldsymbol{h}_{\boldsymbol{i n t}}\right\|_{\mathbf{H}^{k+1 / 2}(\partial \omega)}+\left\|\boldsymbol{h}_{\text {ext }}\right\|_{\mathbf{H}^{1 / 2}(\partial \Omega)}\right) .
\end{aligned}
$$

Proof. The proof of the regularity is similar to the proof of Theorem 2.6. Moreover, if $\nu>c_{b}(\Omega \backslash \bar{\omega})\|\boldsymbol{v}\|_{\mathbf{H}^{1}(\Omega \backslash \bar{\omega})}$, then according to Theorem 2.7. the solution is unique and we have the estimate 2.5. Thus using the local estimate on the solution of the Stokes equations (see for example [6, Appendix A.2]), we obtain by induction the announced estimate.

Remark 2.9. Theorems 2.7 and 2.8 are also true replacing the first equation of the systems (2.4) and 2.6 (i.e. $-\nu \Delta \boldsymbol{u}+(\boldsymbol{v} \cdot \nabla) \boldsymbol{u}+(\boldsymbol{u} \cdot \nabla) \boldsymbol{v}+\nabla p=\boldsymbol{f})$ by

$$
-\nu \Delta \boldsymbol{u}+{ }^{t} \nabla \boldsymbol{v} \boldsymbol{u}-\nabla \boldsymbol{u} \boldsymbol{v}+\nabla p=\boldsymbol{f} .
$$

Remark 2.10. Concerning Theorem 2.8, we can prove the following sharper estimate

$$
\begin{aligned}
\|\varphi \boldsymbol{u}\|_{\mathbf{H}^{k+1}\left(\mathcal{C}^{\prime}\right)}+\|\varphi p\|_{\mathrm{H}^{k}\left(\mathcal{C}^{\prime}\right)} & \\
& \leq C\left(\|\boldsymbol{f}\|_{\mathbf{X}^{-1, k-1}\left(\Omega \backslash \bar{\omega}, \mathcal{C}^{\prime}\right)}+\left\|\boldsymbol{h}_{\boldsymbol{i n t}}\right\|_{\mathbf{H}^{k+1 / 2}(\partial \omega)}+\left\|\boldsymbol{h}_{\boldsymbol{e x \boldsymbol { t }}}\right\|_{\mathbf{H}^{1 / 2}(\partial \Omega)}\right)
\end{aligned}
$$

where $\varphi \in C_{c}^{\infty}(\Omega)$ is a truncation function such that $0 \leq \varphi \leq 1, \varphi \equiv 1$ in $\mathcal{C} \cup \bar{\omega}$ and $\varphi \equiv 0$ in $\Omega \backslash\left(\mathcal{C}^{\prime} \cup \bar{\omega}\right)$. The proof relies on the fact that $(\varphi \boldsymbol{u}, \varphi p)$ solves

$$
\left\{\begin{aligned}
-\nu \Delta(\varphi \boldsymbol{u})+\nabla(\varphi p) & =\tilde{\boldsymbol{f}} & & \text { in } \mathcal{C}^{\prime} \\
\operatorname{div}(\varphi \boldsymbol{u}) & =\varphi g+\boldsymbol{u} \cdot \nabla \varphi & & \text { in } \mathcal{C}^{\prime} \\
\varphi \boldsymbol{u} & =\mathbf{0} & & \text { on } \partial \mathcal{V}^{\prime} \\
\varphi \boldsymbol{u} & =\boldsymbol{h}_{\text {int }} & & \text { on } \partial \omega
\end{aligned}\right.
$$


where $\widetilde{\boldsymbol{f}}:=\varphi \boldsymbol{f}-\nu \boldsymbol{u} \Delta \varphi-2 \nu \nabla \boldsymbol{u} \nabla \varphi+p \nabla \varphi+(\boldsymbol{u} \otimes \nabla \varphi) \boldsymbol{v}-(\varphi \boldsymbol{u} \cdot \nabla) \boldsymbol{v}-(\boldsymbol{v} \cdot \nabla) \varphi \boldsymbol{u}$ (see for instance [16, Theorem IV.5.1] or [6, Appendix A.2]).

\section{The problem setting}

Let $\Omega$ be a bounded connected Lipschitz open subset of $\mathbb{R}^{N}$ (with $N=2$ or $N=3$ ). We denote by $\left\{\Gamma_{i}\right\}_{i=1, \ldots, I}$ the connected components of $\partial \Omega$. Let $\delta>0$ fixed (small). We define

$$
\begin{array}{r}
\mathcal{O}_{\delta}:=\left\{\omega \subset \subset \Omega \text { with a } C^{1,1} \text { boundary such that } \mathrm{d}(x, \partial \Omega)>\delta \forall x \in \omega\right. \\
\text { and such that } \Omega \backslash \bar{\omega} \text { is connected }\} .
\end{array}
$$

Moreover, since we will work using the local regularity of the solutions (see the proof of the differentiability result 5.2 for example), we also need to define $\Omega_{\delta}$ an open set with a $C^{\infty}$ boundary such that

$$
\{x \in \Omega ; \mathrm{d}(x, \partial \Omega)>\delta / 2\} \subset \Omega_{\delta} \subset\{x \in \Omega ; \mathrm{d}(x, \partial \Omega)>\delta / 3\} .
$$

Then, in order to make a shape sensitivity analysis, we define

$$
\boldsymbol{U}:=\left\{\boldsymbol{\theta} \in \mathbf{W}^{2, \infty}\left(\mathbb{R}^{N}\right) ; \operatorname{supp}(\boldsymbol{\theta}) \subset \overline{\Omega_{\delta}}\right\} \quad \text { and } \quad \boldsymbol{U}:=\left\{\boldsymbol{\theta} \in \boldsymbol{U} ;\|\boldsymbol{\theta}\|_{2, \infty}<\min \left(\frac{\delta}{3}, 1\right)\right\}
$$

as the space of admissible deformations. These space permit to perturb only the object $\omega$ and not the fixed domain $\Omega$. Notice that if $\boldsymbol{\theta} \in \mathcal{U}$, then $(\mathbf{I}+\boldsymbol{\theta})$ is a diffeomorphism. For such a $\boldsymbol{\theta} \in \mathcal{U}$ and $\omega \in \mathcal{O}_{\delta}$, we check that $\Omega=(\mathbf{I}+\boldsymbol{\theta})(\Omega)$ and we define the perturbed domain $\omega_{\theta}:=(\mathbf{I}+\boldsymbol{\theta})(\omega)$ which is so that $\omega_{\theta} \in \mathcal{O}_{\delta}$.

Let $O$ be a non-empty open subset of $\partial \Omega$. We assume that there exists an open subset $\widetilde{O} \subset \partial \Omega$ of class $C^{1,1}$ such that $O \subset \subset \widetilde{O}$. Let $\boldsymbol{f}_{\boldsymbol{b}} \in \mathbf{H}^{1 / 2}(O)$ be an admissible boundary measurement and $\boldsymbol{g} \in \mathbf{H}^{1 / 2}(\partial \Omega), \boldsymbol{g} \neq \mathbf{0}$ such that $\boldsymbol{g}_{\mid \widetilde{O}} \in \mathbf{H}^{3 / 2}(\widetilde{O})$ and

$$
\int_{\Gamma_{i}} \boldsymbol{g} \cdot \mathbf{n}=0, \quad \forall i=1, \ldots, I .
$$

The introduction of $\widetilde{O}$ and the regularity of $\boldsymbol{g}_{\mid \widetilde{O}}$ are needed to defined properly the functional $J$ below. This is also necessary to defined properly the adjoint problem (5.7) below (see Remark 5.8).

Let us consider, for $\omega \in \mathcal{O}_{\delta}$, the following overdetermined Navier-Stokes boundary values problem:

$$
\left\{\begin{aligned}
-\nu \Delta \boldsymbol{u}+(\boldsymbol{u} \cdot \nabla) \boldsymbol{u}+\nabla p & =\mathbf{0} & & \text { in } \Omega \backslash \bar{\omega} \\
\operatorname{div} \boldsymbol{u} & =0 & & \text { in } \Omega \backslash \bar{\omega} \\
\boldsymbol{u} & =\boldsymbol{g} & & \text { on } \partial \Omega \\
\boldsymbol{u} & =\mathbf{0} & & \text { on } \partial \omega \\
\nu \partial_{\mathbf{n}} \boldsymbol{u}-p \mathbf{n} & =\boldsymbol{f}_{\boldsymbol{b}} & & \text { on } O,
\end{aligned}\right.
$$

with $\nu>0$ a given constant representing the kinematic viscosity of the liquid.

We suppose here that there exists $\omega^{*} \in \mathcal{O}_{\delta}$ such that (3.2) has a solution. This means that the measurement $\boldsymbol{f}_{\boldsymbol{b}}$ is perfect, that is to say without error. Thus, we consider the following geometric inverse problem:

$$
\text { find } \omega \in \mathcal{O}_{\delta} \text { and a pair }(\boldsymbol{u}, p) \text { which satisfy the overdetermined system (3.2). }
$$


To solve this inverse problem, we consider, for $\omega \in \mathcal{O}_{\delta}$, the least squares functional

$$
J(\omega):=\int_{O} m^{2}\left|\left(\nu \partial_{\mathbf{n}} \boldsymbol{u}(\omega)-p(\omega) \mathbf{n}\right)-\boldsymbol{f}_{\boldsymbol{b}}\right|^{2},
$$

where the function $m \in C_{c}^{\infty}(\partial \Omega)$ is such that $\operatorname{supp}(m)=\bar{O}$ and where the couple $(\boldsymbol{u}(\omega), p(\omega)) \in \mathbf{H}^{1}(\Omega \backslash \bar{\omega}) \times \mathrm{L}^{2}(\Omega \backslash \bar{\omega})$ is a solution of the Navier-Stokes problem

$$
\left\{\begin{array}{rlll}
-\nu \Delta \boldsymbol{u}+(\boldsymbol{u} \cdot \nabla) \boldsymbol{u}+\nabla p & =\mathbf{0} & \text { in } \Omega \backslash \bar{\omega} \\
\operatorname{div} \boldsymbol{u} & =0 & \text { in } \Omega \backslash \bar{\omega} \\
\boldsymbol{u} & =\boldsymbol{g} & & \text { on } \partial \Omega \\
\boldsymbol{u} & =\mathbf{0} & & \text { on } \partial \omega .
\end{array}\right.
$$

Remark 3.1. Notice that using a local regularity result similar to Theorem 2.6, we check that $\left.\left(\nu \partial_{\mathbf{n}} \boldsymbol{u}-p \mathbf{n}\right)\right|_{O} \in \mathbf{H}^{1 / 2}(O)$ (because $(\boldsymbol{u}, p)$ belongs to $\mathbf{H}^{2} \times \mathrm{H}^{1}$ in a neighborhood of $O$, since $\widetilde{O}$ is $\left.C^{1,1}\right)$. Hence, the functional $J$ defined by (3.4) is well defined.

Notice that the existence of a solution of problem (3.5) is guaranteed by Theorem 2.1 since we imposed the compatibility condition (3.1). Note also that the solution is not necessarily unique and then the functional $J$ may be multivalued. To overcome this difficulty, we assume $\nu$ big enough and independent of the object $\omega \in \mathcal{O}_{\delta}$ (see Theorem 2.5 in order to guarantee the uniqueness of the solution of the previous problem (once a normalization condition on the pressure $p$ is imposed): $J$ is then single valued. More precisely, we assume that

$$
\nu>c_{b}(\Omega)\left(2\|\Delta \boldsymbol{G}\|_{\boldsymbol{W}^{\prime}}+\frac{2}{\nu}\|(\boldsymbol{G} \cdot \nabla) \boldsymbol{G}\|_{\boldsymbol{W}^{\prime}}+2\|\boldsymbol{G}\|_{\mathbf{H}^{1}\left(\Omega \backslash \overline{\Omega_{\delta}}\right)}\right)
$$

where $\boldsymbol{W}^{\prime}$ is the dual space of

$$
\boldsymbol{W}:=\left\{\boldsymbol{\varphi} \in \mathbf{H}^{1}\left(\Omega \backslash \overline{\Omega_{\delta}}\right) \operatorname{div} \boldsymbol{\varphi}=0 \text { in } \Omega \backslash \overline{\Omega_{\delta}} \text { and } \boldsymbol{\varphi}=\mathbf{0} \text { on } \partial \Omega\right\},
$$

where, according to [4, Lemma 3.3], $\boldsymbol{G} \in \mathbf{H}^{1}(\Omega)$ satisfies

$$
\boldsymbol{G}=\boldsymbol{g} \text { on } \partial \Omega, \quad \operatorname{div} \boldsymbol{G}=0 \text { in } \Omega \text { and } \boldsymbol{G}=\mathbf{0} \text { in } \Omega_{\delta},
$$

and where

$$
c_{b}(\Omega):=\left\{\begin{array}{ccc}
\frac{|\Omega|^{1 / 2}}{2} & \text { if } & N=2 \\
\frac{2 \sqrt{2}|\Omega|^{1 / 6}}{3} & \text { if } & N=3,
\end{array}\right.
$$

(see [16, Vol. 2, Chap. VIII, page 6]). Moreover, we choose the normalization:

$$
\int_{O} m^{2}\left(\nu \partial_{\mathbf{n}} \boldsymbol{u}-p \mathbf{n}\right) \cdot \mathbf{n}=\int_{O} m^{2} \boldsymbol{f}_{\boldsymbol{b}} \cdot \mathbf{n} .
$$

Hence, according to Theorem 2.5, this solution $(\boldsymbol{u}, p)$ is unique and is referred as the state in the rest of the paper. Notice that the terms in 3.9 have a sense using Remark 3.1

Remark 3.2. The estimate (3.6) implies that

$$
\nu>c_{b}(\Omega \backslash \bar{\omega})\|\boldsymbol{u}\|_{\mathbf{H}^{1}(\Omega \backslash \bar{\omega})},
$$

where $c_{b}(\Omega \backslash \bar{\omega})$ is defined analogously to (3.8). Indeed, if $\nu \geq 2 c_{b}(\Omega)\|\boldsymbol{G}\|_{\mathbf{H}^{1}\left(\Omega \backslash \overline{\Omega_{\delta}}\right)}$, we know that $\|\boldsymbol{u}-\boldsymbol{G}\|_{\mathbf{H}^{1}(\Omega)} \leq \frac{2}{\nu}\|\nu \Delta \boldsymbol{G}-(\boldsymbol{G} \cdot \nabla) \boldsymbol{G}\|_{\boldsymbol{W}^{\prime}}$ (see [25, Ch. II, eq. (1.89)]) and we have obviously $c_{b}(\Omega) \geq c_{b}(\Omega \backslash \bar{\omega})$. Moreover, Inequality (3.6) implies the uniqueness of the solution of all the perturbed problems we will consider in this paper. 
Then, we try to minimize the least squares criterion $J$ :

$$
\omega^{*}=\underset{\omega \in \mathcal{O}_{\delta}}{\operatorname{argmin}} J(\omega) .
$$

Indeed, if $\omega^{*}$ solves the inverse problem (3.3), then $J\left(\omega^{*}\right)=0$ and 3.11) holds. Conversely, if $\omega^{*}$ solves the optimization problem 3.11 with $J\left(\omega^{*}\right)=0$, then it is a solution of (3.3).

The needed functional tools. Let $T>0$, that we will have to fix small. We use the shape calculus introduced by Murat and Simon in [23]. Thus, we consider the function

$$
\phi: t \in[0, T) \mapsto \mathbf{I}+t \boldsymbol{V} \in \mathbf{W}^{2, \infty}\left(\mathbb{R}^{N}\right),
$$

where $\boldsymbol{V} \in \boldsymbol{U}$. Note that for small $t, \phi(t)$ is a diffeomorphism of $\mathbb{R}^{N}$ and that $\phi^{\prime}(0)=\boldsymbol{V}$ vanishes on $\partial \Omega$ and even on the tubular neighborhood $\Omega \backslash \overline{\Omega_{\delta}}$ of $\partial \Omega$. For $t \in[0, T)$, we define $\omega_{t}:=\phi(t)(\omega)$ where $\phi$ is defined by (3.12). For the rest of the paper, we use a subscript " $t$ " to indicate that the quantity is defined on the time $t$ dependent domain. For instance, $\mathbf{n}_{t}$ is the external unit normal of $\Omega \backslash \overline{\omega_{t}}$.

Remark 3.3 (A remark on the nonsingular solutions of the Navier-Stokes problem). All the results are still valid for a nonsingular solution $(\boldsymbol{u}, p)$ of the Navier-Stokes Problem (3.5) (see [17, Ch.IV, §3] for details on this notion). However, without the uniqueness of the solution, the functional $J$ may be multivalued. We then differentiate the functional $J$ for a given nonsingular solution.

We can also notice that, even if we do not assume the uniqueness of the solution of the Navier-Stokes Problem (3.5), the solution of the overdetermined Problem (3.2) is unique. Indeed, let us assume that $\left(\boldsymbol{u}_{j}, p_{j}\right), j=0,1$, are two solutions of problem (3.2) corresponding to $\omega=\omega_{j}$. We know using the identifiability Theorem 4.1 that the overdetermination permits to identify the domain $\omega$. Then, using the unique continuation property (see Corollary 4.3 below), we check, like what is done in the proof of the identifiability Theorem 4.1 in Section 4, that $\boldsymbol{u}:=\boldsymbol{u}_{\mathbf{0}}-\boldsymbol{u}_{\mathbf{1}}=\mathbf{0}$ and $p:=p_{0}-p_{1}=0$ in $\Omega \backslash \bar{\omega}$. In conclusion, the overdetermination of Problem (3.2) permits to identify the obstacle $\omega$ and to select, in the non-uniqueness case, a unique solution.

\section{Identifiability result}

We first state an identifiability result similar to Theorem 1.2 proved in [3] by Alvarez et al.: it states that given a fixed $\boldsymbol{g}$, two different geometries $\omega_{0}$ and $\omega_{1}$ in $\mathcal{O}_{\delta}$ yield two different measures $\boldsymbol{f}_{\boldsymbol{b}_{\mathbf{1}}}$ and $\boldsymbol{f}_{\boldsymbol{b}_{\mathbf{2}}}$. Hence problem (3.3) admits a unique solution. We also refer to [13, Theorem 1.2] proved by Doubova et al.

Theorem 4.1 (Identifiability result). Let $\Omega \subseteq \mathbb{R}^{N}, N=2$ or $N=3$, be a bounded Lipschitz domain, and $O$ be a non-empty open subset of $\partial \Omega$ of class $C^{1,1}$. Let

$$
\omega_{0}, \omega_{1} \in\{\omega \subset \subset \Omega ; \omega \text { is a Lipschitz open set and } \Omega \backslash \bar{\omega} \text { is connected }\}
$$

and $\boldsymbol{g} \in \mathbf{H}^{1 / 2}(\partial \Omega)$ with $\boldsymbol{g} \neq \mathbf{0}$ and $\boldsymbol{g}_{\mid O} \in \mathbf{H}^{3 / 2}(O)$, satisfying the flux condition (3.1). Let $\left(\boldsymbol{u}_{\boldsymbol{j}}, p_{j}\right)$ for $j=0,1$, be a solution of

$$
\left\{\begin{array}{rlll}
-\nu \Delta \boldsymbol{u}_{j}+\left(\boldsymbol{u}_{j} \cdot \nabla\right) \boldsymbol{u}_{j}+\nabla p_{j} & =\mathbf{0} & \text { in } \Omega \backslash \overline{\omega_{j}} \\
\operatorname{div} \boldsymbol{u}_{j} & =0 & \text { in } \Omega \backslash \omega_{j} \\
\boldsymbol{u}_{\boldsymbol{j}} & =\boldsymbol{g} & \text { on } \partial \Omega \\
\boldsymbol{u}_{j} & =\mathbf{0} & & \text { on } \partial \omega_{j} .
\end{array}\right.
$$


Assume that $\left(\boldsymbol{u}_{\boldsymbol{j}}, p_{j}\right)$ are such that

$$
-\nu \partial_{\mathbf{n}} \boldsymbol{u}_{\mathbf{0}}+p_{0} \mathbf{n}=-\nu \partial_{\mathbf{n}} \boldsymbol{u}_{\mathbf{1}}+p_{1} \mathbf{n} \quad \text { on } O .
$$

Then $\omega_{0} \equiv \omega_{1}$.

The proof is adapted from [3, Theorem 1.2]. The main ingredient is the following theorem (see [3, Theorem 2.3]) which is a direct adaptation of the unique continuation property stated in [15]:

Theorem 4.2 (Alvarez et al. in [3]; Fabre, Lebeau in [15]). Let $\Omega_{0} \subset \mathbb{R}^{N}, N \geq 2$, be a connected open set and let $D_{0}$ a non-empty open subset of $\Omega_{0}$. If $\boldsymbol{a} \in \mathbf{L}_{\mathrm{loc}}^{\infty}\left(\Omega_{0}\right)$, $c \in \mathrm{L}_{\mathrm{loc}}^{r}\left(\Omega_{0}, \mathcal{M}_{N, N}\right)$, with $r>N$, and $(\boldsymbol{u}, p) \in \mathbf{H}_{\mathrm{loc}}^{1}\left(\Omega_{0}\right) \times \mathrm{L}_{\text {loc }}^{2}\left(\Omega_{0}\right)$ is a solution of

$$
\left\{\begin{array}{rll}
-\Delta \boldsymbol{u}+(\boldsymbol{a} \cdot \nabla) \boldsymbol{u}+c \boldsymbol{u}+\nabla p & =\mathbf{0} & \text { in } \Omega_{0} \\
\operatorname{div} \boldsymbol{u} & =0 & \text { in } \Omega_{0},
\end{array}\right.
$$

with $\boldsymbol{u}=\mathbf{0}$ in $D_{0}$, then $\boldsymbol{u}=\mathbf{0}$ in $\Omega_{0}$ and $p$ is constant in $\Omega_{0}$.

We deduce from the above theorem the following corollary:

Corollary 4.3 (Unique continuation property). Let $\Omega_{0} \subseteq \mathbb{R}^{N}, N \geq 2$, be a connected open set and $O \subset \partial \Omega_{0}$ be a non-empty Lipschitz relatively open subset. Let us assume that $(\boldsymbol{u}, p) \in \mathbf{H}^{1}\left(\Omega_{0}\right) \times \mathrm{L}^{2}\left(\Omega_{0}\right)$ is a solution of

$$
\left\{\begin{array}{rlll}
-\nu \Delta \boldsymbol{u}+(\boldsymbol{u} \cdot \nabla) \boldsymbol{v}_{\mathbf{1}}+\left(\boldsymbol{v}_{\mathbf{0}} \cdot \nabla\right) \boldsymbol{u}+\nabla p & =\mathbf{0} & \text { in } \Omega_{0} \\
\operatorname{div} \boldsymbol{u} & =0 & \text { in } \Omega_{0} \\
\boldsymbol{u} & =\mathbf{0} & \text { on } O \\
-\nu \partial_{\mathbf{n}} \boldsymbol{u}+p \mathbf{n} & =\mathbf{0} & \text { on } O,
\end{array}\right.
$$

where $\boldsymbol{v}_{\mathbf{0}} \in \mathbf{L}_{\text {loc }}^{\infty}\left(\Omega_{0}\right)$ and $\nabla \boldsymbol{v}_{\mathbf{1}} \in \mathrm{L}_{\mathrm{loc}}^{r}\left(\Omega_{0}, \mathcal{M}_{N, N}\right)$, with $r>N$. Assume also that there exists a neighborhood $\mathcal{V}$ of $O$ in $\bar{\Omega}$ such that $\boldsymbol{v}_{\mathbf{0}} \in \mathbf{L}^{\infty}(\mathcal{V})$ and $\nabla \boldsymbol{v}_{\mathbf{1}} \in \mathrm{L}^{r}\left(\mathcal{V}, \mathcal{M}_{N, N}\right)$. Then $\boldsymbol{u}=\mathbf{0}$ and $p=0$ in $\Omega_{0}$.

Proof of Corollary 4.3. The idea of the proof is to enlarge the domain $\Omega_{0}$ at a part of $O$ and to use the unique continuation Theorem 4.2 in this enlarged domain. We choose $y \in O$ and a radius $\rho \leq \frac{\mathrm{d}(y, \partial O)}{2}\left(\right.$ or $\rho \leq \frac{|\partial \Omega|}{2}$ if $\left.O=\partial \Omega\right)$ such that $\left(\mathcal{B}(y, \rho) \cap \Omega_{0}\right) \subset \mathcal{V}$ and such that $\Omega_{0} \cap \mathcal{B}(y, \rho)$ and $\mathcal{B}(y, \rho) \backslash \overline{\Omega_{0}}$ are Lipschitz (where $\mathcal{B}(y, \rho)$ is the ball with center $y$ and radius $\rho)$. We then set $\Omega_{\rho}:=\Omega_{0} \cup \mathcal{B}(y, \rho)$ and we define

$$
\begin{aligned}
& \widetilde{\boldsymbol{u}}=\left\{\begin{array}{ll}
\boldsymbol{u} & \text { in } \Omega_{0} \\
\mathbf{0} & \text { in } \Omega_{\rho} \backslash \Omega_{0}
\end{array} \quad \text { and } \quad \tilde{p}= \begin{cases}p & \text { in } \Omega_{0} \\
0 & \text { in } \Omega_{\rho} \backslash \Omega_{0},\end{cases} \right. \\
& \widetilde{\boldsymbol{v}_{\mathbf{0}}}=\left\{\begin{aligned}
\boldsymbol{v}_{\mathbf{0}} & \text { in } \Omega_{0} \\
\mathbf{0} & \text { in } \Omega_{\rho} \backslash \Omega_{0}
\end{aligned} \quad \text { and } \quad \widetilde{\nabla \boldsymbol{v}_{\mathbf{1}}}=\left\{\begin{aligned}
\nabla \boldsymbol{v}_{\mathbf{1}} & \text { in } \Omega_{0} \\
0 & \text { in } \Omega_{\rho} \backslash \Omega_{0} .
\end{aligned}\right.\right.
\end{aligned}
$$

Since $\boldsymbol{u} \in \mathbf{H}^{1}\left(\Omega_{0}\right)$ and $\boldsymbol{u}=\mathbf{0}$ on $O$, we check that $\widetilde{\boldsymbol{u}} \in \mathbf{H}^{1}\left(\Omega_{\rho}\right)$. Moreover, $\widetilde{p} \in \mathrm{L}^{2}\left(\Omega_{\rho}\right)$, $\widetilde{\boldsymbol{v}_{0}} \in \mathrm{L}_{\text {loc }}^{\infty}\left(\Omega_{\rho}\right)$ and $\widetilde{\nabla \boldsymbol{v}_{1}} \in \mathrm{L}_{\text {loc }}^{r}\left(\Omega_{\rho}\right)$. By summing on $\Omega_{0} \cap \mathcal{B}(y, \rho)$ and on $\left.\Omega_{\rho}^{\text {ext }}:=\Omega_{\rho}\right\rangle \overline{\Omega_{0}}$ and using the fact that $-\nu \partial_{\mathbf{n}} \boldsymbol{u}+p \mathbf{n}=\mathbf{0}$ on $O$, we get that for all $\boldsymbol{\varphi}$ in $\mathcal{D}(\mathcal{B}(y, \rho))$

$$
\int_{\mathcal{B}(y, \rho)}(\nu \nabla \widetilde{\boldsymbol{u}}: \nabla \boldsymbol{\varphi}-\widetilde{p} \operatorname{div} \boldsymbol{\varphi})+b_{\mathcal{B}(y, \rho)}\left(\widetilde{\boldsymbol{u}}, \widetilde{\boldsymbol{v}_{\mathbf{1}}}, \boldsymbol{\varphi}\right)+b_{\mathcal{B}(y, \rho)}\left(\widetilde{\boldsymbol{v}_{\mathbf{0}}}, \widetilde{\boldsymbol{u}}, \boldsymbol{\varphi}\right)=0
$$

Then, $-\nu \Delta \widetilde{\boldsymbol{u}}+(\widetilde{\boldsymbol{u}} \cdot \nabla) \widetilde{\boldsymbol{v}_{\mathbf{1}}}+\left(\widetilde{\boldsymbol{v}_{\mathbf{0}}} \cdot \nabla\right) \widetilde{\boldsymbol{u}}+\nabla \widetilde{p}=\mathbf{0}$ in $\mathcal{D}^{\prime}(\mathcal{B}(y, \rho))$ and then in $\mathcal{D}^{\prime}\left(\Omega_{\rho}\right)$. Proceeding as above, we also get $\operatorname{div} \widetilde{\boldsymbol{u}}=0$ in $\mathcal{D}^{\prime}\left(\Omega_{\rho}\right)$. Moreover, $\widetilde{\boldsymbol{u}}=\mathbf{0}$ in $\Omega_{\rho}^{\text {ext }} \subset \Omega_{\rho}$. Thus, according to the unique continuation Theorem $4.2, \widetilde{\boldsymbol{u}}=\mathbf{0}$ and $\widetilde{p}$ is constant in $\Omega_{\rho}$. Since $\widetilde{p}=0$ in $\Omega_{\rho}^{\text {ext }}, \widetilde{p}=0$ in $\Omega_{\rho}$ and then $p=0$ in $\Omega_{0}$ by restriction. 
The proof of Theorem 4.1 is split into two steps. Firstly, we use the unique continuation Corollary 4.3 to prove that $\boldsymbol{u}_{\mathbf{0}}=\boldsymbol{u}_{\mathbf{1}}$ and $p_{0}=p_{1}$ in $\Omega \backslash \bar{\omega}$ where $\omega$ is the smallest simply connected open set such that $\left(\omega_{0} \cup \omega_{1}\right) \subset \omega$. Secondly, we proceed by contradiction: we assume $\omega_{0} \neq \omega_{1}$ and we use the unique continuation Theorem 4.2 to prove that $\boldsymbol{u}_{\mathbf{1}}=\mathbf{0}$ (respectively $\boldsymbol{u}_{\mathbf{0}}=\mathbf{0}$ ) on $\partial \Omega$ which contradicts the fact that $\boldsymbol{u}_{\mathbf{1}}=\boldsymbol{g} \neq \mathbf{0}$ (respectively $\left.\boldsymbol{u}_{\mathbf{0}}=\boldsymbol{g} \neq \mathbf{0}\right)$ on $\partial \Omega$.

Proof of Theorem 4.1. We define $\boldsymbol{u}:=\boldsymbol{u}_{\mathbf{0}}-\boldsymbol{u}_{1}, p:=p_{0}-p_{1}$ and let $\omega$ be the smallest simply connected open set such that $\left(\omega_{0} \cup \omega_{1}\right) \subset \omega$ (see Figure 1). Notice that, in the particular

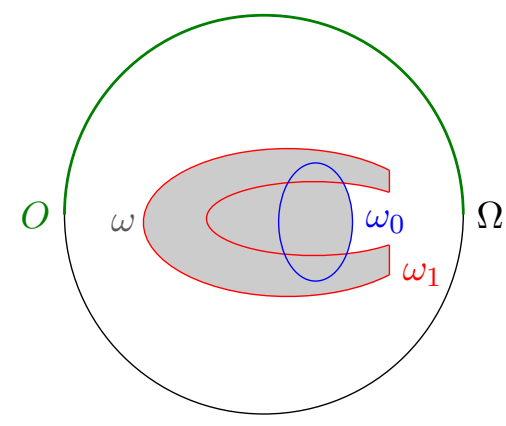

Figure 1: The set $\omega$

case where $\omega_{0} \cup \omega_{1}$ is simply connected, $\omega$ is exactly $\omega_{0} \cup \omega_{1}$. Hence, the pair $(\boldsymbol{u}, p)$ satisfies

$$
\left\{\begin{array}{rlll}
-\nu \Delta \boldsymbol{u}+(\boldsymbol{u} \cdot \nabla) \boldsymbol{u}_{\mathbf{0}}+\left(\boldsymbol{u}_{\mathbf{1}} \cdot \nabla\right) \boldsymbol{u}+\nabla p & =\mathbf{0} & \text { in } \Omega \backslash \bar{\omega} \\
\operatorname{div} \boldsymbol{u} & =0 & \text { in } \Omega \backslash \bar{\omega} \\
\boldsymbol{u} & =\mathbf{0} & \text { on } O \\
-\nu \partial_{\mathbf{n}} \boldsymbol{u}+p \mathbf{n} & =\mathbf{0} & & \text { on } O .
\end{array}\right.
$$

In order to use the previous Corollary 4.3 , we have to check that

$$
\boldsymbol{u}_{\mathbf{1}} \in \mathbf{L}_{\mathrm{loc}}^{\infty}(\Omega \backslash \bar{\omega}) \cap \mathbf{L}^{\infty}(\mathcal{V}), \quad \nabla \boldsymbol{u}_{\mathbf{0}} \in \mathrm{L}_{\mathrm{loc}}^{r}\left(\Omega \backslash \bar{\omega}, \mathcal{M}_{N, N}\right) \cap \mathbf{L}^{r}(\mathcal{V}), \text { with } r>N,
$$

where $\mathcal{V}$ is a neighborhood of $O$ in $\overline{\Omega \backslash \bar{\omega}}$. This comes from a local regularity result similar to Theorem 2.8 since $O$ is $C^{1,1}$ and $\boldsymbol{g}_{\mid O} \in \mathbf{H}^{3 / 2}(O)$, we have, choosing a positive radius $\rho<\mathrm{d}(y, \partial O) / 2$ with $y \in O($ or $\rho<|\partial \Omega| / 2$ if $O=\partial \Omega$ ) in such a way that $\mathcal{B}(y, \rho) \cap \bar{\omega}=\emptyset$ and $\mathcal{B}(y, \rho) \cap\left((\Omega \backslash \bar{\omega})^{c}\right)$ is simply connected,

$$
\boldsymbol{u}_{\mathbf{1}} \in \mathbf{H}^{2}((\Omega \backslash \bar{\omega}) \cap \mathcal{B}(y, \rho)) \hookrightarrow \mathbf{L}^{\infty}((\Omega \backslash \bar{\omega}) \cap \mathcal{B}(y, \rho))
$$

and

$$
\nabla \boldsymbol{u}_{\mathbf{0}} \in \mathrm{H}^{1}\left((\Omega \backslash \bar{\omega}) \cap \mathcal{B}(y, \rho), \mathcal{M}_{N, N}\right) \hookrightarrow \mathrm{L}^{6}\left((\Omega \backslash \bar{\omega}) \cap \mathcal{B}(y, \rho), \mathcal{M}_{N, N}\right) .
$$

Similarly, we prove that $\boldsymbol{u}_{\mathbf{1}} \in \mathbf{L}_{\text {loc }}^{\infty}(\Omega \backslash \bar{\omega})$ and $\nabla \boldsymbol{u}_{\mathbf{0}} \in \mathrm{L}_{\text {loc }}^{r}\left(\Omega \backslash \bar{\omega}, \mathcal{M}_{N, N}\right)$. Therefore, according to Corollary 4.3, $\boldsymbol{u}=\mathbf{0}$ and $p=0$ in $\Omega \backslash \bar{\omega}$.

We then proceed by contradiction assuming that $\omega_{0} \backslash \overline{\omega_{1}}$ is non-empty. In the sequel, we assume that $\omega \backslash \overline{\omega_{1}}$ is connected. If not, it suffices to replace $\omega \backslash \overline{\omega_{1}}$ by one of its connected components in the following proof. We know that

$$
-\nu \Delta \boldsymbol{u}_{1}+\left(\boldsymbol{u}_{\mathbf{1}} \cdot \nabla\right) \boldsymbol{u}_{\mathbf{1}}+\nabla p_{1}=\mathbf{0} \quad \text { in } \omega \backslash \overline{\omega_{1}} .
$$

In order to give a clear idea of the proof, let us first consider the particular case where $\omega=\omega_{0} \cup \omega_{1}$. 
Case where $\omega=\omega_{0} \cup \omega_{1}$. Assume for the moment that $\omega_{0} \backslash \overline{\omega_{1}}$ is Lipschitz. Then, multiplying Equation (4.1) by $\boldsymbol{u}_{1}$ and integrating by parts in $\omega_{0} \backslash \overline{\omega_{1}}$, we obtain, since $\boldsymbol{u}_{\mathbf{1}}=\mathbf{0}$ on $\partial \omega_{1}$,

$$
\int_{\omega_{0} \backslash \overline{\omega_{1}}} \nu\left|\nabla \boldsymbol{u}_{\mathbf{1}}\right|^{2}+\int_{\left(\partial \omega_{0}\right) \backslash \overline{\omega_{1}}}\left(-\nu \partial_{\mathbf{n}} \boldsymbol{u}_{\mathbf{1}}+p_{1} \mathbf{n}\right) \cdot \boldsymbol{u}_{\mathbf{1}}+\frac{1}{2} \int_{\left(\partial \omega_{0}\right) \backslash \overline{\omega_{1}}} \boldsymbol{u}_{\mathbf{1}} \cdot \mathbf{n}\left|\boldsymbol{u}_{\mathbf{1}}\right|^{2}=0 .
$$

Since $\boldsymbol{u}_{\mathbf{0}}=\boldsymbol{u}_{\mathbf{1}}$ in $\Omega \backslash \bar{\omega}$, the boundary condition satisfied by $\boldsymbol{u}_{\mathbf{0}}$ on $\partial \omega_{0}$ provides that $\boldsymbol{u}_{\mathbf{1}}=\boldsymbol{u}_{\mathbf{0}}=\mathbf{0}$ on $\left(\partial \omega_{0}\right) \backslash \overline{\omega_{1}}$. Hence, equality 4.2 is simply

$$
\int_{\omega_{0} \backslash \overline{\omega_{1}}} \nu\left|\nabla \boldsymbol{u}_{1}\right|^{2}=0
$$

Therefore, $\boldsymbol{u}_{\mathbf{1}}$ is constant in $\omega_{0} \backslash \overline{\omega_{1}}$. By Theorem 4.2 (with $c \equiv 0$ ), $\boldsymbol{u}_{\mathbf{1}}$ is then constant in $\Omega \backslash \overline{\omega_{1}}$. Since $\boldsymbol{u}_{\mathbf{1}}=\mathbf{0}$ on $\partial \omega_{1}$, then $\boldsymbol{u}_{\mathbf{1}}=\mathbf{0}$ in $\Omega \backslash \overline{\omega_{1}}$ which contradicts the boundary condition $\boldsymbol{u}_{\mathbf{1}}=\boldsymbol{g} \neq \mathbf{0}$ on $\partial \Omega$. Thus $\omega_{0} \backslash \overline{\omega_{1}}=\emptyset$.

Let us now turn to the general case where $\omega_{0} \backslash \overline{\omega_{1}}$ is not necessary Lipschitz. In such a case, the integration by parts yielding $(4.2)$ is not well justified. Then, to overcome this difficulty and get $(4.3)$, let us use a density argument. We define the space $\widetilde{\mathbf{H}^{1}}\left(\omega_{0} \backslash \overline{\omega_{1}}\right)$ as the space of the functions which belong to $\mathbf{H}^{1}\left(\omega_{0} \backslash \overline{\omega_{1}}\right)$ such that its extension by $\mathbf{0}$ in $\Omega$ belongs to $\mathbf{H}^{1}(\Omega)$, i.e.

$$
\widetilde{\mathbf{H}^{1}}\left(\omega_{0} \backslash \overline{\omega_{1}}\right):=\left\{\boldsymbol{w} \in \mathbf{H}^{1}\left(\omega_{0} \backslash \overline{\omega_{1}}\right), \widetilde{\boldsymbol{w}}:=\left\{\begin{array}{ll}
\boldsymbol{w} & \text { in } \omega_{0} \backslash \overline{\omega_{1}} \\
\mathbf{0} & \text { in } \omega_{1} \\
\mathbf{0} & \text { in } \Omega \backslash \overline{\omega_{0}}
\end{array} \text { belongs to } \mathbf{H}^{1}(\Omega)\right\} .\right.
$$

Since $\partial\left(\omega_{0} \backslash \overline{\omega_{1}}\right)$ is continuous, the space $\mathcal{D}\left(\omega_{0} \backslash \overline{\omega_{1}}\right)$ is dense in $\widetilde{\mathbf{H}^{1}}\left(\omega_{0} \backslash \overline{\omega_{1}}\right)$ according to [18. Theorem 1.4.2.2, page 24] (we also refer to the general definition of the space $\mathbf{H}_{0}^{1}$ given in [19, Definition 3.3.43]). We then multiply (4.1) by $\boldsymbol{\varphi} \in \mathcal{D}\left(\omega_{0} \backslash \overline{\omega_{1}}\right)$ and, using an integration by parts, we get

$$
\int_{\omega_{0} \backslash \overline{\omega_{1}}}\left(\nu \nabla \boldsymbol{u}_{1}: \nabla \boldsymbol{\varphi}-p_{1} \operatorname{div} \boldsymbol{\varphi}\right)+\frac{1}{2} b_{\omega_{0} \backslash \overline{\omega_{1}}}\left(\boldsymbol{u}_{1}, \boldsymbol{u}_{1}, \boldsymbol{\varphi}\right)-\frac{1}{2} b_{\omega_{0} \backslash \overline{\omega_{1}}}\left(\boldsymbol{u}_{1}, \boldsymbol{\varphi}, \boldsymbol{u}_{1}\right)=0,
$$

since $b_{\omega_{0} \backslash \overline{\omega_{1}}}\left(\boldsymbol{u}_{\mathbf{1}}, \boldsymbol{u}_{1}, \boldsymbol{\varphi}\right)=-b_{\omega_{0} \backslash \overline{\omega_{1}}}\left(\boldsymbol{u}_{\mathbf{1}}, \boldsymbol{\varphi}, \boldsymbol{u}_{\mathbf{1}}\right)$. Then it suffices to show that $\boldsymbol{u}_{\mathbf{1}} \mid \omega_{0} \backslash \overline{\omega_{1}}$ belongs to the space $\widetilde{\mathbf{H}^{1}}\left(\omega_{0} \backslash \overline{\omega_{1}}\right)$. Indeed, writing Equality 4.4 with $\varphi=\varphi_{n}$, where $\left(\boldsymbol{\varphi}_{n}\right)_{n \in \mathbb{N}} \subset \mathcal{D}\left(\omega_{0} \backslash \overline{\omega_{1}}\right)$ is a sequence such that $\boldsymbol{\varphi}_{n} \rightarrow \boldsymbol{u}_{\mathbf{1} \mid \omega_{0} \backslash \overline{\omega_{1}}}$ in $\mathbf{H}^{1}\left(\omega_{0} \backslash \overline{\omega_{1}}\right)$, we pass to the limit to get (4.3) and conclude as previously. Hence, let us prove that $\boldsymbol{u}_{1 \mid \omega_{0} \backslash \overline{\omega_{1}}}$ belongs to $\widetilde{\mathbf{H}^{1}}\left(\omega_{0} \backslash \overline{\omega_{1}}\right)$. Then, we define $\widetilde{\boldsymbol{u}_{\mathbf{1}}}$ the extension of $\boldsymbol{u}_{\mathbf{1}}$ by $\mathbf{0}$ in $\omega_{1}$, i.e.

$$
\widetilde{\boldsymbol{u}_{1}}=\left\{\begin{array}{cl}
\boldsymbol{u}_{\mathbf{1}} & \text { in } \Omega \backslash \overline{\omega_{1}} \\
\mathbf{0} & \text { in } \omega_{1}
\end{array}\right.
$$

Since $\boldsymbol{u}_{\mathbf{1}}=\mathbf{0}$ on $\partial \omega_{1}, \widetilde{\boldsymbol{u}_{\mathbf{1}}}$ belongs to $\mathbf{H}^{1}(\Omega)$. We then consider $\left.\widetilde{\boldsymbol{u}_{\mathbf{1}}}\right|_{\omega_{0}} \in \mathbf{H}^{1}\left(\omega_{0}\right)$ and we extend it by $\mathbf{0}$ in $\Omega \backslash \overline{\omega_{0}}$. Since $\widetilde{\boldsymbol{u}}_{\mathbf{1}}=\boldsymbol{u}_{\mathbf{0}}=\mathbf{0}$ on $\partial \omega_{0} \backslash \overline{\omega_{1}}$ and $\widetilde{\boldsymbol{u}}_{\mathbf{1}}=\mathbf{0}$ on $\partial \omega_{0} \cap \omega_{1}$ by construction, this extension, noted again $\widetilde{\boldsymbol{u}_{\mathbf{1}}}$, i.e.

$$
\widetilde{\boldsymbol{u}_{\mathbf{1}}}= \begin{cases}\boldsymbol{u}_{\mathbf{1}} & \text { in } \omega_{0} \backslash \overline{\omega_{1}} \\ \mathbf{0} & \text { in } \omega_{1} \\ \mathbf{0} & \text { in } \Omega \backslash \overline{\omega_{0}}\end{cases}
$$

belongs to $\mathbf{H}^{1}(\Omega)$. Hence $\left.\widetilde{\boldsymbol{u}_{1}}\right|_{\omega_{0} \backslash \overline{\omega_{1}}}=\boldsymbol{u}_{\mathbf{1} \mid \omega_{0} \backslash \overline{\omega_{1}}}$ belongs to $\widetilde{\mathbf{H}^{1}}\left(\omega_{0} \backslash \overline{\omega_{1}}\right)$ and we conlude. 
Case where $\omega \neq \omega_{0} \cup \omega_{1}$ (as in Figure 1 for example). Let us now emphasize the differences and the difficulties of the proof in the general case where $\omega$ is not necessary equal to $\omega_{0} \cup \omega_{1}$. The idea is to replace $\omega_{0}$ by $\omega \backslash \overline{\left(\omega_{1} \backslash \overline{\omega_{0}}\right)}$. Indeed, we can not work with $\omega_{0}$ since we have no information on $\boldsymbol{u}_{\mathbf{1}}$ on $\partial \omega_{0} \backslash \partial \omega$ : we have only proved that $\boldsymbol{u}_{\mathbf{1}}=\boldsymbol{u}_{\mathbf{0}}$ in $\Omega \backslash \bar{\omega}$.

In the case where $\omega \backslash \overline{\omega_{1}}$ is Lipschitz, we proceed in exactly the same way as in the previous Lipschitz case noticing that $\omega_{0} \backslash \overline{\omega_{1}}$ is then replaced by $\omega \backslash \overline{\omega_{1}}$.

In the general case where $\omega \backslash \overline{\omega_{1}}$ is not necessary Lipschitz, we can not prove that $\boldsymbol{u}_{1|\omega| \overline{\omega_{1}}}$ belongs to $\widetilde{\mathbf{H}^{1}}\left(\omega \backslash \overline{\omega_{1}}\right)$ in the same manner than above. Indeed, after having extended $\boldsymbol{u}_{\mathbf{1}}$ by $\mathbf{0}$ in $\omega_{1}$, we can not claim that the extension of $\left.\widetilde{\boldsymbol{u}_{1}}\right|_{\omega \backslash} \overline{\left(\omega_{1} \backslash \overline{\left.\omega_{0}\right)}\right.}$ by $\mathbf{0}$ in $\Omega$ belongs to $\mathbf{H}^{1}(\Omega)$. The idea is then to enlarge the domain $\omega \backslash \overline{\omega_{1}}$ inside $\omega_{1}$ by a smooth (at least Lipschitz) domain $\widetilde{\omega}_{0}$ (see Figure 2). We then make the same proof than in the first case using a

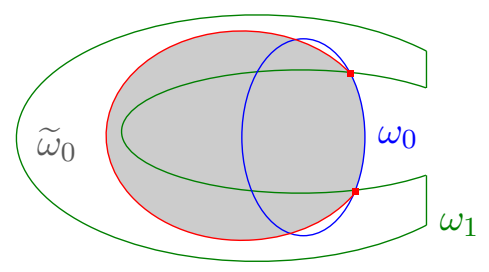

Figure 2: The set $\widetilde{\omega}_{0}$

density argument replacing $\omega_{0}$ by $\widetilde{\omega}_{0}$.

In conclusion, we have proved that $\omega_{0} \backslash \overline{\omega_{1}}=\emptyset$. By symmetry, $\omega_{1} \backslash \overline{\omega_{0}}=\emptyset$ and finally we obtain $\omega_{0}=\omega_{1}$.

\section{$5 \quad$ First order shape analysis and shape derivatives}

\subsection{Differentiability result of the Navier-Stokes problem}

Let $\boldsymbol{\theta} \in \mathcal{U}$. We set $\left(\boldsymbol{u}_{\boldsymbol{\theta}}, p_{\theta}\right) \in \mathbf{H}^{1}\left(\Omega \backslash \overline{\omega_{\theta}}\right) \times \mathrm{L}^{2}\left(\Omega \backslash \overline{\omega_{\theta}}\right)$ solution of

$$
\left\{\begin{array}{rlll}
-\nu \Delta \boldsymbol{u}_{\boldsymbol{\theta}}+\left(\boldsymbol{u}_{\boldsymbol{\theta}} \cdot \nabla\right) \boldsymbol{u}_{\boldsymbol{\theta}}+\nabla p_{\theta} & =\mathbf{0} & \text { in } \Omega \backslash \overline{\omega_{\theta}} \\
\operatorname{div} \boldsymbol{u}_{\boldsymbol{\theta}} & =0 & \text { in } \Omega \backslash \overline{\omega_{\theta}} \\
\boldsymbol{u}_{\boldsymbol{\theta}} & =\boldsymbol{g} & \text { on } \partial \Omega \\
\boldsymbol{u}_{\boldsymbol{\theta}} & =\mathbf{0} & & \text { on } \partial \omega_{\theta},
\end{array}\right.
$$

with

$$
\left\langle m^{2}\left(\nu \partial_{\mathbf{n}} \boldsymbol{u}_{\boldsymbol{\theta}}-p_{\theta} \mathbf{n}\right), \mathbf{n}\right\rangle_{O}=\left\langle m^{2} \boldsymbol{f}_{\boldsymbol{b}}, \mathbf{n}\right\rangle_{O} .
$$

Notice that, since we assumed (3.6), we have, for all $\boldsymbol{\theta} \in \mathcal{U}$,

$$
\nu>c_{b}\left(\Omega \backslash \overline{\omega_{\theta}}\right)\left\|\boldsymbol{u}_{\theta}\right\|_{\mathbf{H}^{1}\left(\Omega \backslash \overline{\omega_{\theta}}\right)},
$$

where $c_{b}\left(\Omega \backslash \overline{\omega_{\theta}}\right)$ is defined analogously to 3.8 (see Remark 3.2 . Hence, using Theorem 2.5 . the pair $\left(\boldsymbol{u}_{\boldsymbol{\theta}}, p_{\theta}\right)$ is unique.

Let $\boldsymbol{G} \in \mathbf{H}^{1}(\Omega)$ satisfying $(3.7)$. Then $\left(\boldsymbol{w}_{\boldsymbol{\theta}}:=\boldsymbol{u}_{\boldsymbol{\theta}}-\boldsymbol{G}, p_{\theta}\right) \in \mathbf{H}_{0}^{1}\left(\Omega \backslash \overline{\omega_{\theta}}\right) \times \mathrm{L}^{2}\left(\Omega \backslash \overline{\omega_{\theta}}\right)$ is 
such that for all $\boldsymbol{\varphi}_{\boldsymbol{\theta}} \in \mathbf{H}_{0}^{1}\left(\Omega \backslash \overline{\omega_{\theta}}\right)$ and for all $\xi_{\theta} \in \mathrm{L}^{2}\left(\Omega \backslash \overline{\omega_{\theta}}\right)$

$$
\left\{\begin{aligned}
\int_{\Omega \backslash \overline{\omega_{\theta}}} \nu \nabla \boldsymbol{w}_{\boldsymbol{\theta}}: \nabla \boldsymbol{\varphi}_{\boldsymbol{\theta}}+b_{\Omega \backslash \overline{\omega_{\theta}}}\left(\boldsymbol{w}_{\boldsymbol{\theta}}, \boldsymbol{w}_{\boldsymbol{\theta}}, \boldsymbol{\varphi}_{\boldsymbol{\theta}}\right)+b_{\Omega \backslash \overline{\omega_{\theta}}}\left(\boldsymbol{w}_{\boldsymbol{\theta}}, \boldsymbol{G}, \boldsymbol{\varphi}_{\boldsymbol{\theta}}\right) & \\
+b_{\Omega \backslash \overline{\omega_{\theta}}}\left(\boldsymbol{G}, \boldsymbol{w}_{\boldsymbol{\theta}}, \boldsymbol{\varphi}_{\boldsymbol{\theta}}\right)-\int_{\Omega \backslash \overline{\omega_{\theta}}} p_{\theta} \operatorname{div} \boldsymbol{\varphi}_{\boldsymbol{\theta}}= & -\int_{\Omega \backslash \overline{\omega_{\theta}}} \nu \nabla \boldsymbol{G}: \nabla \boldsymbol{\varphi}_{\boldsymbol{\theta}} \\
\int_{\Omega \backslash \overline{\omega_{\theta}}} \xi_{\theta} \operatorname{div} \boldsymbol{w}_{\boldsymbol{\theta}}= & -b_{\Omega \backslash \overline{\omega_{\theta}}}\left(\boldsymbol{G}, \boldsymbol{G}, \boldsymbol{\varphi}_{\boldsymbol{\theta}}\right) \\
\left\langle m^{2}\left(\nu \partial_{\mathbf{n}} \boldsymbol{w}_{\boldsymbol{\theta}}-p_{\theta} \mathbf{n}\right), \mathbf{n}\right\rangle_{O}= & \left\langle m^{2} \boldsymbol{f}_{\boldsymbol{b}}, \mathbf{n}\right\rangle_{O} \\
& -\left\langle\nu m^{2} \partial_{\mathbf{n}} \boldsymbol{G}, \mathbf{n}\right\rangle_{O} .
\end{aligned}\right.
$$

Let us define the key objects of our differentiability proof:

$$
\boldsymbol{v}_{\boldsymbol{\theta}}:=\boldsymbol{w}_{\boldsymbol{\theta}} \circ(\mathbf{I}+\boldsymbol{\theta}) \in \mathbf{H}_{0}^{1}(\Omega \backslash \bar{\omega}) \quad \text { and } \quad q_{\theta}:=p_{\theta} \circ(\mathbf{I}+\boldsymbol{\theta}) \in \mathrm{L}^{2}(\Omega \backslash \bar{\omega}) .
$$

To prove that $(\boldsymbol{u}, p)$ is differentiable with respect to the shape, we need the following three lemmas:

Lemma 5.1 (Characterization of $\left.\left(\boldsymbol{v}_{\boldsymbol{\theta}}, q_{\theta}\right)\right)$. For $\boldsymbol{\theta} \in \mathcal{U}$, the pair $\left(\boldsymbol{v}_{\boldsymbol{\theta}}, q_{\theta}\right)$ satisfies for all $\varphi \in \mathbf{H}_{0}^{1}(\Omega \backslash \bar{\omega})$ and all $\xi \in \mathrm{L}^{2}(\Omega \backslash \bar{\omega})$

$$
\left\{\begin{aligned}
\int_{\Omega \backslash \bar{\omega}}\left[\left(\nu \nabla \boldsymbol{v}_{\boldsymbol{\theta}} A(\boldsymbol{\theta})\right): \nabla \boldsymbol{\varphi}-q_{\theta} B(\boldsymbol{\theta}): \nabla \boldsymbol{\varphi}\right] & \\
+b_{J}\left(\boldsymbol{\theta}, \boldsymbol{v}_{\boldsymbol{\theta}}, \boldsymbol{v}_{\boldsymbol{\theta}}, \boldsymbol{\varphi}\right)+b_{\Omega \backslash \bar{\omega}}\left(\boldsymbol{G}, \boldsymbol{v}_{\boldsymbol{\theta}}, \boldsymbol{\varphi}\right) & \\
+b_{\Omega \backslash \bar{\omega}}\left(\boldsymbol{v}_{\boldsymbol{\theta}}, \boldsymbol{G}, \boldsymbol{\varphi}\right) & =-\int_{\Omega \backslash \bar{\omega}} \nu \nabla \boldsymbol{G}: \nabla \boldsymbol{\varphi}-b_{\Omega \backslash \bar{\omega}}(\boldsymbol{G}, \boldsymbol{G}, \boldsymbol{\varphi}) \\
\int_{\Omega \backslash \bar{\omega}}\left(\nabla \boldsymbol{v}_{\boldsymbol{\theta}}: B(\boldsymbol{\theta})\right) \xi & =0 \\
\left\langle m^{2}\left(\nu \partial_{\mathbf{n}} \boldsymbol{v}_{\boldsymbol{\theta}}-q_{\theta} \mathbf{n}\right), \mathbf{n}\right\rangle_{O} & =\left\langle m^{2} \boldsymbol{f}_{\boldsymbol{b}}, \mathbf{n}\right\rangle_{O}-\left\langle\nu m^{2} \partial_{\mathbf{n}} \boldsymbol{G}, \mathbf{n}\right\rangle_{O},
\end{aligned}\right.
$$

with

$$
\begin{aligned}
J_{\boldsymbol{\theta}} & :=\operatorname{det}(\mathrm{I}+\nabla \boldsymbol{\theta}) \in \mathrm{W}^{1, \infty}\left(\overline{\Omega_{\delta}}\right) \\
A(\boldsymbol{\theta}) & :=\nu J_{\boldsymbol{\theta}}(\mathrm{I}+\nabla \boldsymbol{\theta})^{-1}\left(\mathrm{I}+{ }^{t} \nabla \boldsymbol{\theta}\right)^{-1} \in \mathrm{W}^{1, \infty}\left(\overline{\Omega_{\delta}}, \mathcal{M}_{N, N}\right), \\
B(\boldsymbol{\theta}) & :=J_{\boldsymbol{\theta}}\left(\mathrm{I}+{ }^{t} \nabla \boldsymbol{\theta}\right)^{-1} \in \mathrm{W}^{1, \infty}\left(\overline{\Omega_{\delta}}, \mathcal{M}_{N, N}\right), \\
b_{J}(\boldsymbol{\theta}, \boldsymbol{u}, \boldsymbol{v}, \boldsymbol{w}) & :=\sum_{i, j=1}^{N} \int_{\Omega \backslash \bar{\omega}} u_{i}\left[\nabla v_{j} \cdot\left(\partial_{x_{i}}\left((\mathbf{I}+\boldsymbol{\theta})^{-1}\right) \circ(\mathbf{I}+\boldsymbol{\theta})\right)\right] w_{j} J_{\theta} .
\end{aligned}
$$

Lemma 5.2 (Differentiability of $\boldsymbol{\theta} \mapsto\left(\boldsymbol{v}_{\boldsymbol{\theta}}, q_{\theta}\right)$ ). The function

$$
\boldsymbol{\theta} \in \mathcal{U} \mapsto\left(\boldsymbol{v}_{\boldsymbol{\theta}}, q_{\theta}\right) \in \mathbf{X}^{1,2}\left(\Omega \backslash \bar{\omega}, \Omega_{\delta} \backslash \bar{\omega}\right) \times \mathrm{X}^{0,1}\left(\Omega \backslash \bar{\omega}, \Omega_{\delta} \backslash \bar{\omega}\right)
$$

is differentiable in a neighborhood of $\mathbf{0}$ (and even $C^{\infty}$ ).

Remark 5.3. This lemma implies that there exists a constant $c>0$ such that

$$
\left\|\boldsymbol{u}_{\boldsymbol{\theta}} \circ(\mathbf{I}+\boldsymbol{\theta})-\boldsymbol{u}\right\|_{\mathbf{X}^{1,2}\left(\Omega \backslash \bar{\omega}, \Omega_{\delta} \backslash \bar{\omega}\right)}+\left\|p_{\boldsymbol{\theta}} \circ(\mathbf{I}+\boldsymbol{\theta})-p\right\|_{\mathbf{X}^{0,1}\left(\Omega \backslash \bar{\omega}, \Omega_{\delta} \backslash \bar{\omega}\right)} \leq c\|\boldsymbol{\theta}\|_{\mathcal{U}} .
$$

The following lemma is proved in exactly the same way than the analogous Lemma in the Stokes case (see [6, Lemma 3.3]): 
Lemma 5.4 (Differentiability of $\boldsymbol{\theta} \mapsto\left(\boldsymbol{u}_{\boldsymbol{\theta}}, p_{\theta}\right)$ ). There exists $\widetilde{\boldsymbol{u}}_{\boldsymbol{\theta}}, \widetilde{p}_{\theta}$ some respective extensions in $\Omega$ of $\boldsymbol{u}_{\boldsymbol{\theta}} \in \mathbf{H}^{1}(\Omega \backslash \bar{\omega}), p_{\theta} \in \mathrm{L}^{2}(\Omega \backslash \bar{\omega})$ such that the functions

$$
\boldsymbol{\theta} \in \mathcal{U} \mapsto \widetilde{\boldsymbol{u}}_{\boldsymbol{\theta}} \in \mathbf{H}^{1}(\Omega) \quad \text { and } \quad \boldsymbol{\theta} \in \mathcal{U} \mapsto \widetilde{p}_{\theta} \in \mathrm{L}^{2}(\Omega)
$$

are differentiable at $\mathbf{0}$ (and even $C^{1}$ in a neighborhood of $\mathbf{0}$ ).

Proof of Lemma 5.1. The idea is to make the change of variables $x=(\mathbf{I}+\boldsymbol{\theta}) y$ in the variational formulation (5.2). This proof is similar to the proof of Lemma 3.1 in [6] and we refer to this paper for details. We here only focus on the trilinear form $b_{\Omega \backslash \overline{\omega_{\theta}}}(\cdot, \cdot, \cdot)$.

Let $\boldsymbol{\varphi} \in \mathbf{H}_{0}^{1}(\Omega \backslash \bar{\omega})$ and let us define $\boldsymbol{\varphi}_{\boldsymbol{\theta}}:=\boldsymbol{\varphi} \circ(\mathbf{I}+\boldsymbol{\theta})^{-1} \in \mathbf{H}_{0}^{1}\left(\Omega \backslash \overline{\omega_{\theta}}\right)$. We have

$$
\begin{aligned}
b_{\Omega \backslash \overline{\omega_{\theta}}}\left(\boldsymbol{w}_{\boldsymbol{\theta}}, \boldsymbol{w}_{\boldsymbol{\theta}}, \boldsymbol{\varphi}_{\boldsymbol{\theta}}\right) & =\sum_{i, j=1}^{N} \int_{\Omega \backslash \overline{\omega_{\theta}}} w_{\theta}^{i} \frac{\partial w_{\theta}^{j}}{\partial x_{i}} \varphi_{\theta}^{j} \\
& =\sum_{i, j=1}^{N} \int_{\Omega \backslash \overline{\omega_{\theta}}}\left(v_{\theta}^{i} \circ(\mathbf{I}+\boldsymbol{\theta})^{-1}\right)\left[\left(\nabla v_{\theta}^{j} \circ(\mathbf{I}+\boldsymbol{\theta})^{-1}\right) \cdot \frac{\partial\left((\mathbf{I}+\boldsymbol{\theta})^{-1}\right)}{\partial x_{i}}\right] \\
& =\sum_{i, j=1}^{N} \int_{\Omega \backslash \bar{\omega}} v_{\theta}^{i}\left[\nabla v_{\theta}^{j} \cdot\left(\frac{\partial\left((\mathbf{I}+\boldsymbol{\theta})^{-1}\right)}{\partial x_{i}} \circ(\mathbf{I}+\boldsymbol{\theta})\right)\right] \varphi^{j} J_{\theta} .
\end{aligned}
$$

We conclude as in [6, Lemma 3.1] using the fact that $\boldsymbol{\theta} \equiv \mathbf{0}$ in $\Omega \backslash \overline{\Omega_{\delta}}$ and $\boldsymbol{G} \equiv \mathbf{0}$ in $\Omega_{\delta}$.

The following proof use weighted Sobolev spaces in order to take into account of the local regularity of the solutions in a neighborhood of the objects. This proof establishes a mathematical framework to study the existence of the shape derivatives in this kind of problem with an intuitive idea: the regularity of the exterior boundary does not have importance.

Proof of Lemma 5.2. In this proof, we use the classical implicit functions theorem.

Step 1: notations. We need some additional tools: a fourth domain $\widetilde{\Omega}_{\delta}$ which is an open set with a $C^{\infty}$ boundary such that $\Omega_{\delta} \subset \subset \widetilde{\Omega}_{\delta} \subset \subset \Omega$ and a truncation function $\Phi \in C_{c}^{\infty}\left(\widetilde{\Omega}_{\delta}\right)$ such that $\Phi \equiv 1$ in $\Omega_{\delta}$. We define the spaces:

$$
\begin{aligned}
& \boldsymbol{E}_{\mathbf{1}}:=\left\{(\boldsymbol{v}, q) \in \mathbf{H}_{0}^{1}(\Omega \backslash \bar{\omega}) \times \mathrm{L}^{2}(\Omega \backslash \bar{\omega}) ;(\Phi \boldsymbol{v}, \Phi q) \in \mathbf{H}^{2}(\Omega \backslash \bar{\omega}) \times \mathrm{H}^{1}(\Omega \backslash \bar{\omega})\right\}, \\
& \boldsymbol{E}_{\mathbf{2}}:=\left\{\boldsymbol{f} \in \mathbf{H}^{-1}(\Omega \backslash \bar{\omega}) ; \Phi \boldsymbol{f} \in \mathbf{L}^{2}(\Omega \backslash \bar{\omega})\right\}, \\
& E_{3}:=\left\{\psi \in \mathrm{L}^{2}(\Omega \backslash \bar{\omega}) ; \Phi \psi \in \mathrm{H}^{1}(\Omega \backslash \bar{\omega}), \int_{\Omega \backslash \bar{\omega}} \psi=0\right\} .
\end{aligned}
$$

Note that $\boldsymbol{E}_{\mathbf{1}}, \boldsymbol{E}_{\mathbf{2}}$ and $E_{3}$ are Hilbert spaces with respective norms

$$
\begin{aligned}
\|(\boldsymbol{v}, q)\|_{\boldsymbol{E}_{1}}^{2} & :=\|\boldsymbol{v}\|_{\mathbf{H}^{1}(\Omega \backslash \bar{\omega})}^{2}+\|q\|_{\mathrm{L}^{2}(\Omega \backslash \bar{\omega})}^{2}+\|\Phi \boldsymbol{v}\|_{\mathbf{H}^{2}(\Omega \backslash \bar{\omega})}^{2}+\|\Phi q\|_{\mathrm{H}^{1}(\Omega \backslash \bar{\omega})}^{2}, \\
\|\boldsymbol{f}\|_{\boldsymbol{E}_{\mathbf{2}}}^{2} & :=\|\boldsymbol{f}\|_{\mathbf{H}^{-1}(\Omega \backslash \bar{\omega})}^{2}+\|\Phi \boldsymbol{f}\|_{\mathbf{L}^{2}(\Omega \backslash \bar{\omega})}^{2}, \\
\|\psi\|_{E_{3}}^{2} & :=\|\psi\|_{\mathrm{L}^{2}(\Omega \backslash \bar{\omega})}^{2}+\|\Phi \psi\|_{\mathrm{H}^{1}(\Omega \backslash \bar{\omega})}^{2} .
\end{aligned}
$$

Moreover, we have $\boldsymbol{E}_{\mathbf{1}} \hookrightarrow \mathbf{X}^{1,2}\left(\Omega \backslash \bar{\omega}, \Omega_{\delta} \backslash \bar{\omega}\right) \times \mathrm{X}^{0,1}\left(\Omega \backslash \bar{\omega}, \Omega_{\delta} \backslash \bar{\omega}\right), \boldsymbol{E}_{\mathbf{2}} \hookrightarrow \mathbf{X}^{-1,0}\left(\Omega \backslash \bar{\omega}, \Omega_{\delta} \backslash \bar{\omega}\right)$ and $E_{3} \hookrightarrow \mathrm{X}^{0,1}\left(\Omega \backslash \bar{\omega}, \Omega_{\delta} \backslash \bar{\omega}\right)$. Using the notations introduced in Lemma 5.1, we also define: 
- $\boldsymbol{F}_{\mathbf{1}}(\boldsymbol{\theta},(\boldsymbol{v}, q)) \in \mathbf{H}^{-1}(\Omega \backslash \bar{\omega})$ by $\forall \boldsymbol{\varphi} \in \mathbf{H}_{0}^{1}(\Omega \backslash \bar{\omega})$,

$$
\left\langle\boldsymbol{F}_{\mathbf{1}}(\boldsymbol{\theta},(\boldsymbol{v}, q)), \boldsymbol{\varphi}\right\rangle_{\Omega \backslash \bar{\omega}}:=\int_{\Omega \backslash \bar{\omega}}[(\nu \nabla v A(\boldsymbol{\theta})): \nabla \varphi-q B(\boldsymbol{\theta}): \nabla \boldsymbol{\varphi}],
$$

- $\boldsymbol{F}_{\mathbf{2}}(\boldsymbol{\theta},(\boldsymbol{v}, q)) \in \mathbf{H}^{-1}(\Omega \backslash \bar{\omega})$ by $\forall \boldsymbol{\varphi} \in \mathbf{H}_{0}^{1}(\Omega \backslash \bar{\omega})$,

$$
\left\langle\boldsymbol{F}_{\mathbf{2}}(\boldsymbol{\theta},(\boldsymbol{v}, q)), \boldsymbol{\varphi}\right\rangle_{\Omega \backslash \bar{\omega}}:=b_{J}(\boldsymbol{\theta}, \boldsymbol{v}, \boldsymbol{v}, \boldsymbol{\varphi})+b_{\Omega \backslash \bar{\omega}}(\boldsymbol{v}, \boldsymbol{G}, \boldsymbol{\varphi})+b_{\Omega \backslash \bar{\omega}}(\boldsymbol{G}, \boldsymbol{v}, \boldsymbol{\varphi}),
$$

- $\boldsymbol{F}_{\mathbf{3}} \in \mathbf{H}^{-1}(\Omega \backslash \bar{\omega})$ by $\forall \boldsymbol{\varphi} \in \mathbf{H}_{0}^{1}(\Omega \backslash \bar{\omega})$,

$$
\left\langle\boldsymbol{F}_{\mathbf{3}}, \boldsymbol{\varphi}\right\rangle_{\Omega \backslash \bar{\omega}}:=\int_{\Omega \backslash \bar{\omega}} \nu \nabla \boldsymbol{G}: \nabla \boldsymbol{\varphi}+b_{\Omega \backslash \bar{\omega}}(\boldsymbol{G}, \boldsymbol{G}, \boldsymbol{\varphi}),
$$

- $\overline{\boldsymbol{F}}(\boldsymbol{\theta},(\boldsymbol{v}, q)) \in \mathbf{H}^{-1}(\Omega \backslash \bar{\omega})$ by $\forall \boldsymbol{\varphi} \in \mathbf{H}_{0}^{1}(\Omega \backslash \bar{\omega})$,

$$
\langle\overline{\boldsymbol{F}}(\boldsymbol{\theta},(\boldsymbol{v}, q)), \boldsymbol{\varphi}\rangle_{\Omega \backslash \bar{\omega}}:=\left\langle\boldsymbol{F}_{\mathbf{1}}(\boldsymbol{\theta},(\boldsymbol{v}, q))+\boldsymbol{F}_{\mathbf{2}}(\boldsymbol{\theta},(\boldsymbol{v}, q))+\boldsymbol{F}_{\mathbf{3}}, \boldsymbol{\varphi}\right\rangle_{\Omega \backslash \bar{\omega}},
$$

- $\boldsymbol{F}: \mathcal{U} \times \boldsymbol{E}_{\mathbf{1}} \rightarrow \boldsymbol{E}_{\mathbf{2}} \times E_{3} \times \mathbb{R}$ by

$$
\boldsymbol{F}(\boldsymbol{\theta},(\boldsymbol{v}, q))=\left(\overline{\boldsymbol{F}}(\boldsymbol{\theta},(\boldsymbol{v}, q)), \nabla \boldsymbol{v}: B(\boldsymbol{\theta}),\left\langle m^{2}\left(\nu \partial_{\mathbf{n}} \boldsymbol{v}-q \mathbf{n}-\boldsymbol{f}_{\boldsymbol{b}}+\nu \partial_{\mathbf{n}} \boldsymbol{G}\right), \mathbf{n}\right\rangle_{O}\right) .
$$

Note that $\boldsymbol{F}$ is well-defined. In particular $\nabla \boldsymbol{v}: B(\boldsymbol{\theta})$ belongs to $E_{3}$ because, using the change of variables $x=(\mathbf{I}+\boldsymbol{\theta})^{-1} y$, we check that

$$
\int_{\Omega \backslash \bar{\omega}} \nabla \boldsymbol{v}: B(\boldsymbol{\theta})=\int_{\Omega \backslash \overline{\bar{\omega}_{\theta}}} \operatorname{div}\left(\boldsymbol{v} \circ(\mathbf{I}+\boldsymbol{\theta})^{-1}\right)=0,
$$

since $\boldsymbol{v} \in \mathbf{H}_{0}^{1}(\Omega \backslash \bar{\omega})$ (and then $\left.\boldsymbol{v} \circ(\mathbf{I}+\boldsymbol{\theta})^{-1} \in \mathbf{H}_{0}^{1}\left(\Omega \backslash \overline{\omega_{\theta}}\right)\right)$.

Step 2: for $\boldsymbol{\theta} \equiv \mathbf{0}$. We have $\boldsymbol{F}\left(\mathbf{0},\left(\boldsymbol{v}_{\mathbf{0}}, q_{0}\right)\right)=\boldsymbol{F}(\mathbf{0},(\boldsymbol{w}, p))=(\mathbf{0}, 0,0)$.

Step 3: differentiability of $\boldsymbol{F}$. In the same way as what is done in the Stokes case in [6], we prove that

$$
(\boldsymbol{\theta},(\boldsymbol{v}, q)) \in \mathcal{U} \times \boldsymbol{E}_{\mathbf{1}} \mapsto \overline{\boldsymbol{F}}(\boldsymbol{\theta},(\boldsymbol{v}, q)) \in \boldsymbol{E}_{\mathbf{2}} \quad \text { and } \quad(\boldsymbol{\theta},(\boldsymbol{v}, q)) \in \mathcal{U} \times \boldsymbol{E}_{\mathbf{1}} \mapsto \nabla \boldsymbol{v}: B(\boldsymbol{\theta}) \in E_{3}
$$

are $C^{\infty}$. Moreover, the function $(\boldsymbol{v}, q) \in \boldsymbol{E}_{\mathbf{1}} \mapsto\left\langle m^{2}\left(\nu \partial_{\mathbf{n}} \boldsymbol{v}-q \mathbf{n}-\boldsymbol{f}_{\boldsymbol{b}}+\nu \partial_{\mathbf{n}} \boldsymbol{G}\right), \mathbf{n}\right\rangle_{O} \in \mathbb{R}$ is $C^{\infty}$. We then conclude that $\boldsymbol{F}$ is $C^{\infty}$.

Step 4: $\mathrm{D}_{(\boldsymbol{w}, p)} \boldsymbol{F}(\mathbf{0},(\boldsymbol{w}, p))$ is an isomorphism. In order to simplify the notations, we use $\boldsymbol{w}$ for $\boldsymbol{w}_{\mathbf{0}}$ in the end of this proof. We compute for all $(\boldsymbol{v}, q) \in \boldsymbol{E}_{\mathbf{1}}$

$$
\begin{aligned}
\mathrm{D}_{(\boldsymbol{w}, p)} \boldsymbol{F}(\mathbf{0},(\boldsymbol{w}, p))(\boldsymbol{v}, q)=(-\nu \Delta \boldsymbol{v}+(\boldsymbol{v} \cdot \nabla) \boldsymbol{w}+(\boldsymbol{w} \cdot \nabla) \boldsymbol{v} & +\nabla q, \operatorname{div} \boldsymbol{v}, \\
& \left.\left\langle m^{2}\left(\nu \partial_{\mathbf{n}} \boldsymbol{v}-q \mathbf{n}\right), \mathbf{n}\right\rangle_{O}\right) .
\end{aligned}
$$

Let $(\boldsymbol{\chi}, \eta, r) \in \boldsymbol{E}_{\mathbf{2}} \times E_{3} \times \mathbb{R}$. Let us prove there exists a unique pair $(\boldsymbol{v}, q) \in \boldsymbol{E}_{\mathbf{1}}$ such that

$$
\left\{\begin{array}{rlrlrl}
-\nu \Delta \boldsymbol{v}+(\boldsymbol{v} \cdot \nabla) \boldsymbol{w}+(\boldsymbol{w} \cdot \nabla) \boldsymbol{v}+\nabla q & = & & \text { in } \Omega \backslash \bar{\omega} \\
\operatorname{div} \boldsymbol{v} & =\eta & & \text { in } \Omega \backslash \bar{\omega} \\
\boldsymbol{v} & = & \mathbf{0} & \text { on } \partial \Omega \\
\boldsymbol{v} & =\mathbf{0} & & \text { on } \partial \omega \\
\left\langle m^{2}\left(\nu \partial_{\mathbf{n}} \boldsymbol{v}-q \mathbf{n}\right), \mathbf{n}\right\rangle_{O} & = & & &
\end{array}\right.
$$


Note that the compatibility condition $\int_{\Omega \backslash \bar{\omega}} \eta=0$ is satisfied because $\eta \in E_{3}$. Moreover, since $\nu>c_{b}(\Omega \backslash \bar{\omega})\|\boldsymbol{w}\|_{\mathbf{H}^{1}(\Omega \backslash \bar{\omega})}$ by assumption (3.10) (see also Remark 3.2), Theorem 2.7 guarantees that there exists a unique solution $(\boldsymbol{v}, q) \in \mathbf{H}_{0}^{1}(\Omega \backslash \bar{\omega}) \times \mathrm{L}^{2}(\Omega \backslash \bar{\omega})$ of Problem $\sqrt{5.3}$ and the local regularity Theorem 2.8 proves $(\boldsymbol{v}, q)$ belongs to $\mathbf{H}^{2}\left(\Omega_{\delta} \backslash \bar{\omega}\right) \times \mathrm{H}^{1}\left(\Omega_{\delta} \backslash \bar{\omega}\right)$. More precisely, we obtain

$$
(\Phi \boldsymbol{v}, \Phi q) \in \mathbf{H}^{2}(\Omega \backslash \bar{\omega}) \times \mathrm{H}^{1}(\Omega \backslash \bar{\omega}),
$$

by multiplying $(5.3)$ by $\Phi$ (see Remark 2.10$)$. Note that assumptions of Theorem 2.7 are satisfied since $\boldsymbol{w}_{\mid \Omega_{\delta} \backslash \bar{\omega}}=\boldsymbol{u}_{\mid \Omega_{\delta} \backslash \bar{\omega}} \in \mathbf{H}^{2}\left(\Omega_{\delta} \backslash \bar{\omega}\right)$ by the local regularity Theorem 2.6

Step 5: conclusion. The implicit function theorem applies and we know that there exists a $C^{\infty}$ function $\boldsymbol{\theta} \in \mathcal{U} \mapsto(\boldsymbol{v}(\boldsymbol{\theta}), q(\boldsymbol{\theta})) \in \boldsymbol{E}_{\mathbf{1}}$ such that $\boldsymbol{F}(\boldsymbol{\theta},(\boldsymbol{v}(\boldsymbol{\theta}), q(\boldsymbol{\theta})))=(\mathbf{0}, 0,0)$ in a neighborhood of $\mathbf{0}$. Using the uniqueness of the solution of such a problem, we conclude.

\subsection{First order shape derivatives}

First order shape derivatives of the state. We aim to make a sensitivity (with respect to the shape) analysis. The Navier-Stokes problem on $\Omega \backslash \overline{\omega_{t}}$

$$
\left\{\begin{array}{rlll}
-\nu \Delta \boldsymbol{u}_{\boldsymbol{t}}+\left(\boldsymbol{u}_{\boldsymbol{t}} \cdot \nabla\right) \boldsymbol{u}_{\boldsymbol{t}}+\nabla p_{t} & =\mathbf{0} & & \text { in } \Omega \backslash \overline{\omega_{t}} \\
\operatorname{div} \boldsymbol{u}_{\boldsymbol{t}} & =0 & \text { in } \Omega \backslash \bar{\omega}_{t} \\
\boldsymbol{u}_{\boldsymbol{t}} & =\boldsymbol{g} & & \text { on } \partial \Omega \\
\boldsymbol{u}_{\boldsymbol{t}} & =\mathbf{0} & & \text { on } \partial \omega_{t},
\end{array}\right.
$$

admits a solution $\left(\boldsymbol{u}_{\boldsymbol{t}}, p_{t}\right) \in \mathbf{H}^{1}\left(\Omega \backslash \overline{\omega_{t}}\right) \times \mathrm{L}^{2}\left(\Omega \backslash \overline{\omega_{t}}\right)$ satisfying the normalization condition

$$
\int_{O} m^{2}\left(\nu \partial_{\mathbf{n}} \boldsymbol{u}_{t}-p_{t} \mathbf{n}\right) \cdot \mathbf{n}=\int_{O} m^{2} \boldsymbol{f}_{\boldsymbol{b}} \cdot \mathbf{n} .
$$

To be more clear, let us remain the definition of the shape derivative in our situation (see [19] for details).

- If the mapping $\boldsymbol{\theta} \in \mathcal{U} \mapsto\left(\boldsymbol{u}_{\boldsymbol{\theta}} \circ(\mathbf{I}+\boldsymbol{\theta}), p_{\boldsymbol{\theta}} \circ(\mathbf{I}+\boldsymbol{\theta})\right) \in \mathbf{H}^{1}(\Omega \backslash \bar{\omega}) \times \mathrm{L}^{2}(\Omega \backslash \bar{\omega})$ is Fréchet differentiable at $\mathbf{0}$, we say that $\boldsymbol{\theta} \mapsto\left(\boldsymbol{u}_{\boldsymbol{\theta}}, p_{\boldsymbol{\theta}}\right)$ possesses a total first variation (or derivative) at $\mathbf{0}$. In such a case, this total first derivative at $\mathbf{0}$ in the direction $\boldsymbol{\theta}$ is denoted by $\left(\dot{\boldsymbol{u}}_{\boldsymbol{\theta}}, \dot{p}_{\boldsymbol{\theta}}\right)$.

- If, for every $\mathscr{D} \subset \subset \Omega \backslash \bar{\omega}$, the mapping $\boldsymbol{\theta} \in \mathcal{U} \mapsto\left(\boldsymbol{u}_{\boldsymbol{\theta} \mid \mathscr{D}}, p_{\boldsymbol{\theta} \mid \mathscr{D}}\right) \in \mathbf{H}^{1}(\mathscr{D}) \times \mathrm{L}^{2}(\mathscr{D})$ is Fréchet differentiable at $\mathbf{0}$, we say that $\boldsymbol{\theta} \mapsto\left(\boldsymbol{u}_{\boldsymbol{\theta}}, p_{\boldsymbol{\theta}}\right)$ possesses a local first variation (or derivative) at $\mathbf{0}$. In such a case, this local first derivative at $\mathbf{0}$ in the direction $\boldsymbol{\theta}$ is denoted by $\left(\boldsymbol{u}_{\boldsymbol{\theta}}^{\prime}, p_{\boldsymbol{\theta}}^{\prime}\right)$ and is well defined in the whole domain $\Omega \backslash \bar{\omega}$ :

$$
\left(\boldsymbol{u}_{\boldsymbol{\theta}}^{\prime}, p_{\boldsymbol{\theta}}^{\prime}\right)=\left.\frac{d}{d t}\left(\boldsymbol{u}_{t \boldsymbol{\theta} \mid \mathscr{D}}, p_{t \boldsymbol{\theta} \mid \mathscr{D}}\right)\right|_{t=0} \quad \text { in each } \mathscr{D} \subset \subset \Omega \backslash \bar{\omega} .
$$

We define similarly the higher order shape derivative. In the following, for $\boldsymbol{V} \in \mathcal{U}$, we denote by $\left(\boldsymbol{u}^{\prime}, p^{\prime}\right)$ the local first variation $\left(\boldsymbol{u}_{\boldsymbol{V}}^{\prime}, p_{\boldsymbol{V}}^{\prime}\right)$ which is referred as the shape derivative of the state.

Remark 5.5. Using the same argument than in Remark 3.1, we check that $\left.\left(\nu \partial_{\mathbf{n}} \boldsymbol{u}_{t}-p_{t} \mathbf{n}\right)\right|_{O}$ belongs to $\mathbf{H}^{1 / 2}(O)$. Thus, these two previous terms have a sense. We use again this argument to define the following normalization condition. 
Proposition 5.6 (First order shape derivative of the state). Let $\boldsymbol{V} \in \mathcal{U}$. The solution $(\boldsymbol{u}, p)$ of Problem (3.5) is differentiable with respect to the domain $\omega \in \mathcal{O}_{\delta}$. The shape derivative $\left(\boldsymbol{u}^{\prime}, p^{\prime}\right)$ of $(\boldsymbol{u}, p)$ which belongs to $\mathbf{H}^{1}(\Omega \backslash \bar{\omega}) \times \mathrm{L}^{2}(\Omega \backslash \bar{\omega})$ is the only solution of the following boundary values problem

$$
\left\{\begin{aligned}
-\nu \Delta \boldsymbol{u}^{\prime}+\left(\boldsymbol{u}^{\prime} \cdot \nabla\right) \boldsymbol{u}+(\boldsymbol{u} \cdot \nabla) \boldsymbol{u}^{\prime}+\nabla p^{\prime} & =\mathbf{0} & & \text { in } \Omega \backslash \bar{\omega} \\
\operatorname{div} \boldsymbol{u}^{\prime} & =0 & & \text { in } \Omega \backslash \bar{\omega} \\
\boldsymbol{u}^{\prime} & =\mathbf{0} & & \text { on } \partial \Omega \\
\boldsymbol{u}^{\prime} & =-\partial_{\mathbf{n}} \boldsymbol{u}(\boldsymbol{V} \cdot \mathbf{n}) & & \text { on } \partial \omega
\end{aligned}\right.
$$

with the normalization condition

$$
\left\langle m^{2}\left(\nu \partial_{\mathbf{n}} \boldsymbol{u}^{\prime}-p^{\prime} \mathbf{n}\right), \mathbf{n}\right\rangle_{O}=0 .
$$

Let $\left(\boldsymbol{u}_{\boldsymbol{t}}, p_{t}\right) \in \mathbf{H}^{1}\left(\Omega \backslash \overline{\omega_{t}}\right) \times \mathrm{L}^{2}\left(\Omega \backslash \overline{\omega_{t}}\right)$ be the solution of the perturbed Navier-Stokes problem (5.4). Then

$$
\left\{\begin{array}{rlrl}
\int_{\Omega \backslash \overline{\omega_{t}}}\left\{\nu \nabla \boldsymbol{u}_{\boldsymbol{t}}: \nabla \boldsymbol{\varphi}_{\boldsymbol{t}}-p_{t} \operatorname{div} \boldsymbol{\varphi}_{\boldsymbol{t}}\right\}+b_{\Omega \backslash \overline{\omega_{t}}}\left(\boldsymbol{u}_{\boldsymbol{t}}, \boldsymbol{u}_{\boldsymbol{t}}, \boldsymbol{\varphi}_{t}\right) & =\mathbf{0} & & \forall \boldsymbol{\varphi}_{\boldsymbol{t}} \in \mathbf{H}^{1}\left(\Omega \backslash \overline{\omega_{t}}\right) \\
\int_{\Omega \backslash \overline{\omega_{t}}} \xi_{t} \operatorname{div} \boldsymbol{u}_{\boldsymbol{t}} & =0 & & \forall \xi_{t} \in \mathrm{L}^{2}\left(\Omega \backslash \overline{\omega_{t}}\right) \\
\int_{O} m^{2}\left(\nu \partial_{\mathbf{n}} \boldsymbol{u}_{\boldsymbol{t}}-p_{t} \mathbf{n}\right) \cdot \mathbf{n} & =\int_{O} m^{2} \boldsymbol{f}_{\boldsymbol{b}} \cdot \mathbf{n} . &
\end{array}\right.
$$

Proof of Proposition 5.6. First step: first order shape differentiability. Using the Fréchet differentiability Lemma 5.4 , we obtain the Gâteaux differentiability in the direction $\boldsymbol{V}$ : there exist $\boldsymbol{u}_{\boldsymbol{t}}$ and $p_{t}$, respective extensions in $\Omega$ of $\boldsymbol{u}_{\boldsymbol{t}}$ and $p_{t}$ such that the functions, defined on $[0, T), t \mapsto \boldsymbol{u}_{\boldsymbol{t}} \in \mathbf{H}^{1}(\Omega)$ and $t \mapsto p_{t} \in \mathrm{L}^{2}(\Omega)$ are differentiable at 0 by composition. We denote by $\boldsymbol{u}^{\prime}$ and $p^{\prime}$ their respective derivative at 0 .

Second step: derivative of the normalization condition. The function

$$
\int_{O} m^{2}\left(\nu \partial_{\mathbf{n}} \boldsymbol{u}_{t}-p_{t} \mathbf{n}\right) \cdot \mathbf{n}=\int_{O} m^{2} \boldsymbol{f}_{\boldsymbol{b}} \cdot \mathbf{n}
$$

is constant on $[0, T)$. Thus, its derivative is 0 and we get $\int_{O} m^{2}\left(\nu \partial_{\mathbf{n}} \boldsymbol{u}^{\prime}-p^{\prime} \mathbf{n}\right) \cdot \mathbf{n}=0$.

Third step: derivative of the equalities on $\Omega \backslash \overline{\omega_{t}}$. Let $\varphi \in \mathcal{D}(\Omega \backslash \bar{\omega})$. As $\Omega \backslash \bar{\omega}$ is open, we have, for t small enough, $\varphi \in \mathcal{D}\left(\Omega \backslash \overline{\omega_{t}}\right)$. Therefore, using the first line of the variational formulation (5.6), we have for all $t \in[0, T)$

$$
\begin{aligned}
0 & =\int_{\Omega \backslash \overline{\omega_{t}}}\left(\nu \nabla \boldsymbol{u}_{\boldsymbol{t}}: \nabla \boldsymbol{\varphi}-p_{t} \operatorname{div} \boldsymbol{\varphi}\right)+b_{\Omega \backslash \overline{\omega_{t}}}\left(\boldsymbol{u}_{\boldsymbol{t}}, \boldsymbol{u}_{\boldsymbol{t}}, \boldsymbol{\varphi}\right) \\
& =\int_{\Omega \backslash \bar{\omega}}\left(\nu \nabla \boldsymbol{u}_{t}: \nabla \boldsymbol{\varphi}-p_{t} \operatorname{div} \boldsymbol{\varphi}\right)+b_{\Omega \backslash \bar{\omega}}\left(\boldsymbol{u}_{\boldsymbol{t}}, \boldsymbol{u}_{\boldsymbol{t}}, \boldsymbol{\varphi}\right)
\end{aligned}
$$

We differentiate with respect to $t$ at $t=0$ the equality

$$
0=\int_{\Omega \backslash \bar{\omega}}\left(\nu \nabla \boldsymbol{u}_{\boldsymbol{t}}: \nabla \boldsymbol{\varphi}-p_{t} \operatorname{div} \boldsymbol{\varphi}\right)+b_{\Omega \backslash \bar{\omega}}\left(\boldsymbol{u}_{\boldsymbol{t}}, \boldsymbol{u}_{\boldsymbol{t}}, \boldsymbol{\varphi}\right)
$$

to obtain

$$
\begin{aligned}
0 & =\int_{\Omega \backslash \bar{\omega}}\left(\nu \nabla \boldsymbol{u}^{\prime}: \nabla \boldsymbol{\varphi}-p^{\prime} \operatorname{div} \boldsymbol{\varphi}\right)+b_{\Omega \backslash \bar{\omega}}\left(\boldsymbol{u}^{\prime}, \boldsymbol{u}, \boldsymbol{\varphi}\right)+b_{\Omega \backslash \bar{\omega}}\left(\boldsymbol{u}, \boldsymbol{u}^{\prime}, \boldsymbol{\varphi}\right) \\
& =\left\langle-\nu \Delta \boldsymbol{u}^{\prime}+\left(\boldsymbol{u}^{\prime} \cdot \nabla\right) \boldsymbol{u}+(\boldsymbol{u} \cdot \nabla) \boldsymbol{u}^{\prime}+\nabla p^{\prime}, \boldsymbol{\varphi}\right\rangle_{\mathcal{D}^{\prime}(\Omega \backslash \bar{\omega}), \mathcal{D}(\Omega \backslash \bar{\omega})}
\end{aligned}
$$


As it is true for all $\varphi \in \mathcal{D}(\Omega \backslash \bar{\omega})$, we get

$$
-\nu \Delta \boldsymbol{u}^{\prime}+\left(\boldsymbol{u}^{\prime} \cdot \nabla\right) \boldsymbol{u}+(\boldsymbol{u} \cdot \nabla) \boldsymbol{u}^{\prime}+\nabla p^{\prime}=\mathbf{0} \quad \text { in } \mathcal{D}^{\prime}(\Omega \backslash \bar{\omega}) .
$$

Proceeding with the second line of (5.6) as previously, we check that $\operatorname{div} \boldsymbol{u}^{\prime}=0$ in $\mathcal{D}^{\prime}(\Omega \backslash \bar{\omega})$.

Fourth step: derivatives of the boundary values condition. We differentiate with respect to $t$ the relation $\boldsymbol{u}_{\boldsymbol{t}}=\boldsymbol{g}$ on $\partial \Omega$, at $t=0$. We obtain on $\partial \Omega$ that $\boldsymbol{u}^{\prime}=\mathbf{0}$.

Let us set $\boldsymbol{v}_{\boldsymbol{t}}:=\boldsymbol{u}_{\boldsymbol{t}} \circ \phi(t)$. By differentiation with respect to $t$ (see [19, Corollary 5.2.5]), we obtain $\boldsymbol{u}^{\prime}=\boldsymbol{v}^{\prime}-\nabla \boldsymbol{u} \boldsymbol{V}$ in $\Omega_{\delta} \backslash \bar{\omega}$. Since $\boldsymbol{v}_{\boldsymbol{t}}=\mathbf{0}$ on $\partial \omega$ for all $t \in[0, T), \boldsymbol{v}^{\prime}=\mathbf{0}$ on $\partial \omega$ and $\boldsymbol{u}^{\prime}=-\nabla \boldsymbol{u} \boldsymbol{V}$. Finally, since $\boldsymbol{u}=\mathbf{0}$ on $\partial \omega, \nabla \boldsymbol{u}=\partial_{\mathbf{n}} \boldsymbol{u} \otimes \mathbf{n}$, where $\otimes$ is the tensorial product, and $\boldsymbol{u}^{\prime}=-\partial_{\mathbf{n}} \boldsymbol{u}(\boldsymbol{V} \cdot \mathbf{n})$.

First order shape derivatives of the functional. By introducing an adjoint problem, we then characterize the gradient of the least squares functional $J$ defined by (3.4) in the following proposition.

Proposition 5.7 (First order shape derivative of the functional). For $\boldsymbol{V}$ in $\boldsymbol{U}$, the least squares functional $J$ is differentiable at $\omega$ in the direction $\boldsymbol{V}$ with

$$
\mathrm{D} J(\omega) \cdot \boldsymbol{V}=-\int_{\partial \omega}\left[\left(\nu \partial_{\mathbf{n}} \boldsymbol{w}-\pi \mathbf{n}\right) \cdot \partial_{\mathbf{n}} \boldsymbol{u}\right](\boldsymbol{V} \cdot \mathbf{n}),
$$

where $(\boldsymbol{w}, \pi) \in \mathbf{H}^{1}(\Omega \backslash \bar{\omega}) \times \mathrm{L}^{2}(\Omega \backslash \bar{\omega})$ is the solution of the following boundary values problem:

$$
\left\{\begin{aligned}
-\nu \Delta \boldsymbol{w}+{ }^{t} \nabla \boldsymbol{u} \boldsymbol{w}-\nabla \boldsymbol{w} \boldsymbol{u}+\nabla \pi & =\mathbf{0} & & \text { in } \Omega \backslash \bar{\omega} \\
\operatorname{div} \boldsymbol{w} & =0 & & \text { in } \Omega \backslash \bar{\omega} \\
\boldsymbol{w} & =2 m^{2}\left(\nu \partial_{\mathbf{n}} \boldsymbol{u}-p \mathbf{n}-\boldsymbol{f}_{\boldsymbol{b}}\right) & & \text { on } \partial \Omega \\
\boldsymbol{w} & =\mathbf{0} & & \text { on } \partial \omega
\end{aligned}\right.
$$

with the normalization condition

$$
\left\langle m^{2}\left(\nu \partial_{\mathbf{n}} \boldsymbol{w}-\pi \mathbf{n}\right), \mathbf{n}\right\rangle_{O}=0 .
$$

Remark 5.8. Note that the previous characterization of the gradient is well defined. Indeed, using a local regularity argument similar to Theorem 2.6, we check $\left.\left(\nu \partial_{\mathbf{n}} \boldsymbol{u}-p \mathbf{n}\right)\right|_{O}$ belongs to $\mathbf{H}^{1 / 2}(O)$. Then the boundary condition $2 m^{2}\left(\nu \partial_{\mathbf{n}} \boldsymbol{u}-p \mathbf{n}-\boldsymbol{f}_{\boldsymbol{b}}\right)$ belongs to $\mathbf{H}^{1 / 2}(\partial \Omega)$. Notice also using the local regularity Theorems 2.6 and 2.8 that $\partial_{\mathbf{n}} \boldsymbol{u}$ and $\nu \partial_{\mathbf{n}} \boldsymbol{w}-\pi \mathbf{n}$ belong to $\mathbf{H}^{3 / 2}(\partial \omega)$.

Define, for all $t \in[0, T)$,

$$
J\left(\omega_{t}\right):=j(t):=\int_{O} m^{2}\left|\left(\nu \partial_{\mathbf{n}} \boldsymbol{u}_{t}-p_{t} \mathbf{n}\right)-\boldsymbol{f}_{\boldsymbol{b}}\right|^{2} .
$$

Proof of Proposition 5.7. First step: derivative of $j$ and adjoint problem. By Proposition 5.6. $(\boldsymbol{u}, p)$ is differentiable with respect to the shape. We denote their respective derivative by $\boldsymbol{u}^{\prime}$ and $p^{\prime}$. Differentiating $j$ with respect to $t$ at $t=0$, we obtain

$$
j^{\prime}(0)=2 \int_{O} m^{2}\left(\nu \partial_{\mathbf{n}} \boldsymbol{u}^{\prime}-p^{\prime} \mathbf{n}\right) \cdot\left(\left(\nu \partial_{\mathbf{n}} \boldsymbol{u}-p \mathbf{n}\right)-\boldsymbol{f}_{\boldsymbol{b}}\right) .
$$

Then, we consider the adjoint problem (5.7). According to Theorem 2.7, it admits a unique solution $(\boldsymbol{w}, \pi) \in \mathbf{H}^{1}(\Omega \backslash \bar{\omega}) \times \mathrm{L}^{2}(\Omega \backslash \bar{\omega})$ with $\left\langle m^{2}\left(\nu \partial_{\mathbf{n}} \boldsymbol{w}-\pi \mathbf{n}\right), \mathbf{n}\right\rangle_{O}=0$. Indeed, 
the compatibility condition $\int_{O} m^{2}\left(\nu \partial_{\mathbf{n}} \boldsymbol{u}-p \mathbf{n}-\boldsymbol{f}_{\boldsymbol{b}}\right) \cdot \mathbf{n}=0$ concerning the adjoint problem is true as consequence of our choice of compatibility condition for the state (3.9).

Second step: writing of $j^{\prime}(0)$ as an integral on $\partial \omega$. We proceed by successive integrations by parts. We multiply the first equation of the adjoint problem (5.7) by $\boldsymbol{u}^{\prime}$ to get:

$$
\int_{\Omega \backslash \bar{\omega}} \nu \nabla \boldsymbol{w}: \nabla \boldsymbol{u}^{\prime}+b_{\Omega \backslash \bar{\omega}}\left(\boldsymbol{u}^{\prime}, \boldsymbol{u}, \boldsymbol{w}\right)-b_{\Omega \backslash \bar{\omega}}\left(\boldsymbol{u}, \boldsymbol{w}, \boldsymbol{u}^{\prime}\right)=-\left\langle-\nu \partial_{\mathbf{n}} \boldsymbol{w}+\pi \mathbf{n}, \boldsymbol{u}^{\prime}\right\rangle_{\partial(\Omega \backslash \bar{\omega})},
$$

(since $\operatorname{div} \boldsymbol{u}^{\prime}=0$ in $\Omega \backslash \bar{\omega}$ ). Then, we multiply the first equation of (5.5) by $\boldsymbol{w}$ to obtain

$$
\int_{\Omega \backslash \bar{\omega}} \nu \nabla \boldsymbol{u}^{\prime}: \nabla \boldsymbol{w}+b_{\Omega \backslash \bar{\omega}}\left(\boldsymbol{u}^{\prime}, \boldsymbol{u}, \boldsymbol{w}\right)+b_{\Omega \backslash \bar{\omega}}\left(\boldsymbol{u}, \boldsymbol{u}^{\prime}, \boldsymbol{w}\right)=-\left\langle-\nu \partial_{\mathbf{n}} \boldsymbol{u}^{\prime}+p^{\prime} \mathbf{n}, \boldsymbol{w}\right\rangle_{\partial(\Omega \backslash \bar{\omega})},
$$

(since $\operatorname{div} \boldsymbol{w}=0$ in $\Omega \backslash \bar{\omega})$. Therefore, gathering $(5.8)$ and $(5.9)$ and using the boundary conditions of $\left(\boldsymbol{u}^{\prime}, p^{\prime}\right)$ and $(\boldsymbol{w}, \pi)$ (see Problems (5.5) and (5.7)) and the fact that $b_{\Omega \backslash \bar{\omega}}\left(\boldsymbol{u}, \boldsymbol{w}, \boldsymbol{u}^{\prime}\right)=-b_{\Omega \backslash \bar{\omega}}\left(\boldsymbol{u}, \boldsymbol{u}^{\prime}, \boldsymbol{w}\right)$, we obtain

$$
2 \int_{O} m^{2}\left(\nu \partial_{\mathbf{n}} \boldsymbol{u}^{\prime}-p^{\prime} \mathbf{n}\right) \cdot\left(\left(\nu \partial_{\mathbf{n}} \boldsymbol{u}-p \mathbf{n}\right)-\boldsymbol{f}_{\boldsymbol{b}}\right)=-\int_{\partial \omega}\left(\nu \partial_{\mathbf{n}} \boldsymbol{w}-\pi \mathbf{n}\right) \cdot \partial_{\mathbf{n}} \boldsymbol{u}(\boldsymbol{V} \cdot \mathbf{n}) .
$$

Remark 5.9. Note that for $\omega^{*} \in \mathcal{O}_{\delta}$ solution of the inverse problem (3.3), the boundary condition on $\partial \Omega$ of the adjoint problem (5.7) is homogeneous and then $\boldsymbol{w}=\mathbf{0}$ and $\pi=0$. Hence, we check that for all $\boldsymbol{V} \in \boldsymbol{U}, \mathrm{D} J\left(\omega^{*}\right) \cdot \boldsymbol{V}=0$

\subsection{Differentiability result for the linearized Navier-Stokes equation}

To differentiate the least squares functional, we introduce the adjoint Problem (5.7). By Theorem 2.7, this problem admits a unique solution $(\boldsymbol{w}, \pi) \in \mathbf{H}^{1}(\Omega \backslash \bar{\omega}) \times \mathrm{L}^{2}(\Omega \backslash \bar{\omega})$ with the normalization condition:

$$
\left\langle m^{2}\left(\nu \partial_{\mathbf{n}} \boldsymbol{w}-\pi \mathbf{n}\right), \mathbf{n}\right\rangle_{O}=0,
$$

since we assume that $\nu$ satisfies (3.10). In the same way as we defined Problem (5.1), we defined the adjoint problem on $\Omega \backslash \overline{\omega_{\theta}}$ and we denote by $\left(\boldsymbol{w}_{\boldsymbol{\theta}}, \pi_{\theta}\right)$ its solution such that

$$
\left\langle m^{2}\left(\nu \partial_{\mathbf{n}} \boldsymbol{w}_{\boldsymbol{\theta}}-\pi_{\theta} \mathbf{n}\right), \mathbf{n}\right\rangle_{O}=0 .
$$

According to [4, Lemma 3.3], we consider $\boldsymbol{H} \in \mathbf{H}^{1}(\Omega)$ such that

$$
\boldsymbol{H}=\nu \partial_{\mathbf{n}} \boldsymbol{u}-p \mathbf{n}-\boldsymbol{f}_{\boldsymbol{b}} \text { on } \partial \Omega, \quad \operatorname{div} \boldsymbol{H}=0 \text { in } \Omega \quad \text { and } \boldsymbol{H}=\mathbf{0} \text { in } \Omega_{\delta} .
$$

Thus $\left(\boldsymbol{z}_{\boldsymbol{\theta}}:=\boldsymbol{w}_{\boldsymbol{\theta}}-\boldsymbol{H}, \pi_{\theta}\right) \in \mathbf{H}_{0}^{1}\left(\Omega \backslash \overline{\omega_{\theta}}\right) \times \mathrm{L}^{2}\left(\Omega \backslash \overline{\omega_{\theta}}\right)$ is such that for all $\boldsymbol{\varphi}_{\boldsymbol{\theta}} \in \mathbf{H}_{0}^{1}\left(\Omega \backslash \overline{\omega_{\theta}}\right)$ and for all $\xi_{\theta} \in \mathrm{L}^{2}\left(\Omega \backslash \overline{\omega_{\theta}}\right)$

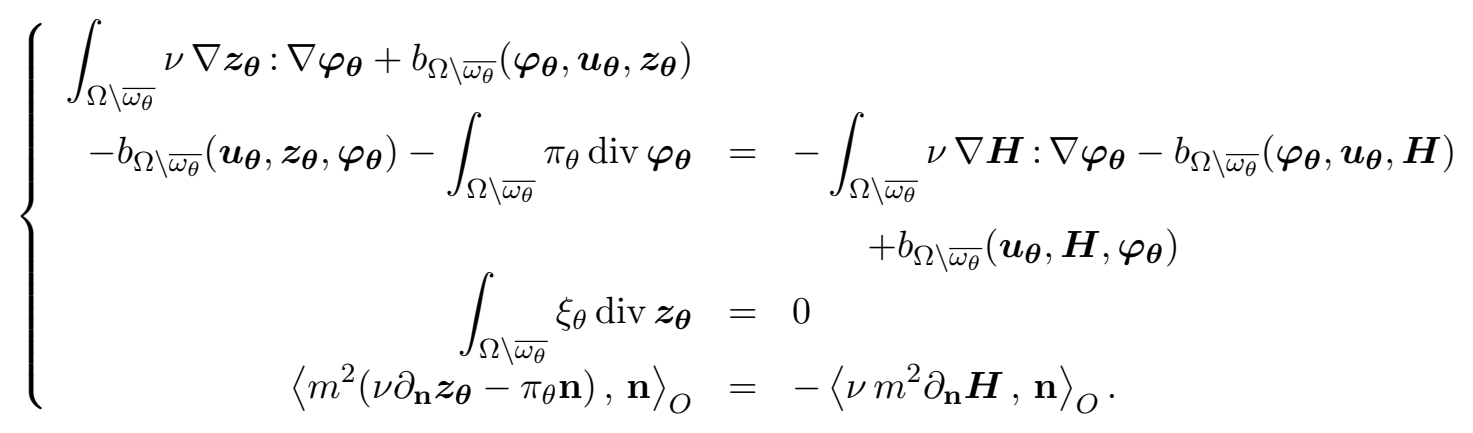

We then proceed in the same way as the proof of the differentiability of the state $(\boldsymbol{u}, p)$ to prove the following lemma: 
Lemma 5.10 (Differentiability of $\boldsymbol{\theta} \mapsto\left(\boldsymbol{w}_{\boldsymbol{\theta}}, \pi_{\theta}\right)$ ). There exists $\widetilde{\boldsymbol{w}}_{\boldsymbol{\theta}}, \widetilde{\pi}_{\theta}$ some respective extensions of $\boldsymbol{w}_{\boldsymbol{\theta}} \in \mathbf{H}^{1}(\Omega \backslash \bar{\omega}), \pi_{\theta} \in \mathrm{L}^{2}(\Omega \backslash \bar{\omega})$ such that the functions

$$
\boldsymbol{\theta} \in \mathcal{U} \mapsto \widetilde{\boldsymbol{w}}_{\boldsymbol{\theta}} \in \mathbf{H}^{1}(\Omega) \quad \text { and } \quad \boldsymbol{\theta} \in \mathcal{U} \mapsto \widetilde{\pi}_{\theta} \in \mathrm{L}^{2}(\Omega)
$$

are differentiable at $\mathbf{0}$. In particular, the solution $(\boldsymbol{w}, \pi)$ is differentiable with respect to the domain.

We precise that, defining $\boldsymbol{F}$ in a similar way as in the proof of Lemma 5.2 , we here have

$$
\begin{aligned}
& \mathrm{D}_{\left(\boldsymbol{z}_{\mathbf{0}}, \pi\right)} \boldsymbol{F}\left(\mathbf{0},\left(\boldsymbol{z}_{\mathbf{0}}, \pi\right)\right)(\boldsymbol{v}, q)=\left(-\nu \Delta \boldsymbol{v}+{ }^{t} \nabla \boldsymbol{z}_{\mathbf{0}} \boldsymbol{v}-\nabla \boldsymbol{v} \boldsymbol{z}_{\mathbf{0}}+\nabla q, \operatorname{div} \boldsymbol{v},\right. \\
&\left.\left\langle m^{2}\left(\nu \partial_{\mathbf{n}} \boldsymbol{v}-q \mathbf{n}\right), \mathbf{n}\right\rangle_{O}\right) .
\end{aligned}
$$

We then use Remark 2.9.

\section{Second order shape analysis and shape derivatives}

\subsection{Differentiability result of the Navier-Stokes problem}

In order to study the second order differentiability, we have to assume more regularity of the object $\omega$ and of the perturbation $\boldsymbol{V}$. Then, we redefine the following tools for the rest of the paper:

$$
\begin{aligned}
& \mathcal{O}_{\delta}:=\left\{\omega \subset \subset \Omega \text { with a } C^{2,1} \text { boundary such that } \mathrm{d}(x, \partial \Omega)>\delta \forall x \in \omega\right. \\
& \text { and such that } \Omega \backslash \bar{\omega} \text { is connected }\}, \\
& \boldsymbol{U}:=\left\{\boldsymbol{\theta} \in \mathbf{W}^{3, \infty}\left(\mathbb{R}^{N}\right) ; \operatorname{supp}(\boldsymbol{\theta}) \subset \overline{\Omega_{\delta}}\right\} \quad \text { and } \boldsymbol{\mathcal { U }}:=\left\{\boldsymbol{\theta} \in \boldsymbol{U} ;\|\boldsymbol{\theta}\|_{3, \infty}<\min \left(\frac{\delta}{3}, 1\right)\right\}(.6 .
\end{aligned}
$$

Obvious adaptation of the proof of the first order differentiability (see Lemma 5.2) with

$$
\begin{aligned}
& \boldsymbol{E}_{\mathbf{1}}:=\left\{(\boldsymbol{v}, q) \in \mathbf{H}_{0}^{1}(\Omega \backslash \bar{\omega}) \times \mathrm{L}^{2}(\Omega \backslash \bar{\omega}) ;(\Phi \boldsymbol{v}, \Phi q) \in \mathbf{H}^{3}(\Omega \backslash \bar{\omega}) \times \mathrm{H}^{2}(\Omega \backslash \bar{\omega})\right\}, \\
& \boldsymbol{E}_{\mathbf{2}}:=\left\{\boldsymbol{f} \in \mathbf{H}^{-1}(\Omega \backslash \bar{\omega}) ; \Phi \boldsymbol{f} \in \mathbf{H}^{1}(\Omega \backslash \bar{\omega})\right\} \\
& E_{3}:=\left\{\psi \in \mathrm{L}^{2}(\Omega \backslash \bar{\omega}), \Phi \psi \in \mathrm{H}^{2}(\Omega \backslash \bar{\omega}), \int_{\Omega \backslash \bar{\omega}} \psi=0\right\}
\end{aligned}
$$

permits to prove the following lemma which is used to check that $(\boldsymbol{u}, p)$ is twice differentiable with respect to the shape:

Lemma 6.1 (Higher order differentiability of $\boldsymbol{\theta} \mapsto\left(\boldsymbol{v}_{\boldsymbol{\theta}}, q_{\theta}\right)$ ). The function

$$
\boldsymbol{\theta} \in \mathcal{U} \mapsto\left(\boldsymbol{v}_{\boldsymbol{\theta}}, q_{\theta}\right) \in \mathbf{X}^{1,3}\left(\Omega \backslash \bar{\omega}, \Omega_{\delta} \backslash \bar{\omega}\right) \times \mathrm{X}^{0,2}\left(\Omega \backslash \bar{\omega}, \Omega_{\delta} \backslash \bar{\omega}\right)
$$

is twice differentiable in a neighborhood of $\mathbf{0}$ (and even $C^{\infty}$ ).

Remark 6.2. This lemma implies that there exists a constant $c>0$ such that

$$
\left\|\boldsymbol{u}_{\boldsymbol{\theta}} \circ(\mathbf{I}+\boldsymbol{\theta})-\boldsymbol{u}\right\|_{\mathbf{X}^{1,3}\left(\Omega \backslash \bar{\omega}, \Omega_{\delta} \backslash \bar{\omega}\right)}+\left\|p_{\boldsymbol{\theta}} \circ(\mathbf{I}+\boldsymbol{\theta})-p\right\|_{\mathbf{X}^{0,2}\left(\Omega \backslash \bar{\omega}, \Omega_{\delta} \backslash \bar{\omega}\right)} \leq c\|\boldsymbol{\theta}\|_{\mathcal{U}} .
$$

We then prove the following lemma in exactly the same way than the analogous Lemma in the Stokes case (see [6, Lemma 3.5]).

Lemma 6.3 (Second order shape differentiability). The solution $(\boldsymbol{u}, p)$ is twice differentiable with respect to the domain. 


\subsection{Second order shape derivatives}

We recall that we work now with more regularity concerning the object $\omega$ and the perturbation $\boldsymbol{V}$. The definitions of $\mathcal{O}_{\delta}, \boldsymbol{U}$ and $\mathcal{U}$ are now given by 6.1) and 6.2). Let us consider $\omega^{*} \in \mathcal{O}_{\delta}$ solution of the inverse problem $(3.3)$ in order to study the stability of the optimization problem (3.11) at $\omega^{*}$.

Proposition 6.4 (Characterization of the shape Hessian at a critical shape). The solutions $(\boldsymbol{u}, p)$ and $(\boldsymbol{w}, \pi)$ are respectively twice and once differentiable with respect to the domain. Moreover, for $\boldsymbol{V} \in \boldsymbol{U}$, we have

$$
\mathrm{D}^{2} J\left(\omega^{*}\right) \cdot \boldsymbol{V} \cdot \boldsymbol{V}=-\int_{\partial \omega^{*}}\left[\left(\nu \partial_{\mathbf{n}} \boldsymbol{w}^{\prime}-\pi^{\prime} \mathbf{n}\right) \cdot \partial_{\mathbf{n}} \boldsymbol{u}\right](\boldsymbol{V} \cdot \mathbf{n}),
$$

where $\left(\boldsymbol{w}^{\prime}, \pi^{\prime}\right) \in \mathbf{H}^{1}\left(\Omega \backslash \overline{\omega^{*}}\right) \times \mathrm{L}^{2}\left(\Omega \backslash \overline{\omega^{*}}\right)$ is the solution of the following problem:

$$
\left\{\begin{aligned}
-\nu \Delta \boldsymbol{w}^{\prime}+{ }^{t} \nabla \boldsymbol{u} \boldsymbol{w}^{\prime}-\nabla \boldsymbol{w}^{\prime} \boldsymbol{u}+\nabla \pi^{\prime} & =\mathbf{0} & & \text { in } \Omega \backslash \overline{\omega^{*}} \\
\operatorname{div} \boldsymbol{w}^{\prime} & =0 & & \text { in } \Omega \backslash \bar{\omega}^{*} \\
\boldsymbol{w}^{\prime} & =2 m^{2}\left(\nu \partial_{\mathbf{n}} \boldsymbol{u}^{\prime}-p^{\prime} \mathbf{n}\right) & & \text { on } \partial \Omega \\
\boldsymbol{w}^{\prime} & =\mathbf{0} & & \text { on } \partial \omega^{*},
\end{aligned}\right.
$$

with the normalization condition

$$
\left\langle m^{2}\left(\nu \partial_{\mathbf{n}} \boldsymbol{w}^{\prime}-\pi^{\prime} \mathbf{n}\right), \mathbf{n}\right\rangle_{O}=0 .
$$

Remark 6.5. Note that $2 m^{2}\left(\nu \partial_{\mathbf{n}} \boldsymbol{u}^{\prime}-p^{\prime} \mathbf{n}-\boldsymbol{f}_{\boldsymbol{b}}\right)$ belongs to $\mathbf{H}^{1 / 2}(\partial \Omega)$ and $\mathrm{D}^{2} J\left(\omega^{*}\right) \cdot \boldsymbol{V} \cdot \boldsymbol{V}$ is well defined.

Notice that, thanks to the local regularity results 2.6 and 2.8

$$
(\boldsymbol{u}, p) \in \mathbf{H}^{3}\left(\Omega_{\delta} \backslash \overline{\omega^{*}}\right) \times \mathrm{H}^{2}\left(\Omega_{\delta} \backslash \overline{\omega^{*}}\right) \quad \text { and } \quad\left(\boldsymbol{u}^{\prime}, p^{\prime}\right) \in \mathbf{H}^{2}\left(\Omega_{\delta} \backslash \overline{\omega^{*}}\right) \times \mathrm{H}^{1}\left(\Omega_{\delta} \backslash \overline{\omega^{*}}\right) .
$$

Proof of Proposition 6.4. First step: second order shape differentiability. According to Lemma 6.3. $(\boldsymbol{u}, p)$ is twice differentiable with respect to the domain. We denote by $\left(\boldsymbol{u}^{\prime \prime}, p^{\prime \prime}\right)$ the second order shape derivatives.

Second step: second derivative of $j$ and derivative of the adjoint problem. Consider $\boldsymbol{V} \in \boldsymbol{U}$. We differentiate the function $j$ twice with respect to $t$. At $t=0$, it holds

$$
j^{\prime \prime}(0)=\mathrm{D}^{2} J(\omega) \cdot \boldsymbol{V} \cdot \boldsymbol{V}=2 \int_{O} m^{2}\left[\left(\nu \partial_{\mathbf{n}} \boldsymbol{u}^{\prime \prime}-p^{\prime \prime} \mathbf{n}\right) \cdot\left(\nu \partial_{\mathbf{n}} \boldsymbol{u}-p \mathbf{n}-\boldsymbol{f}_{\boldsymbol{b}}\right)+\left|\nu \partial_{\mathbf{n}} \boldsymbol{u}^{\prime}-p^{\prime} \mathbf{n}\right|^{2}\right] .
$$

Since $\omega^{*}$ solves the inverse problem, $\nu \partial_{\mathbf{n}} \boldsymbol{u}-p \mathbf{n}=\boldsymbol{f}_{\boldsymbol{b}}$ on $O$. Therefore

$$
\mathrm{D}^{2} J\left(\omega^{*}\right) \cdot \boldsymbol{V} \cdot \boldsymbol{V}=2 \int_{O} m^{2}\left|\nu \partial_{\mathbf{n}} \boldsymbol{u}^{\prime}-p^{\prime} \mathbf{n}\right|^{2}
$$

We introduce $(\boldsymbol{w}, \pi) \in \mathbf{H}^{1}(\Omega \backslash \bar{\omega}) \times \mathrm{L}^{2}(\Omega \backslash \bar{\omega})$ with $\left\langle m^{2}\left(\nu \partial_{\mathbf{n}} \boldsymbol{w}-\pi \mathbf{n}\right), \mathbf{n}\right\rangle_{O}=0$ the solution of the adjoint system (5.7). According to Lemma 5.10, $(\boldsymbol{w}, \pi)$ is differentiable with respect to the domain and we denote $\left(\boldsymbol{w}^{\prime}, \pi^{\prime}\right)$ its shape derivative. In the same manner that we characterized $\boldsymbol{u}^{\prime}$ and $p^{\prime}$ (see Proposition 5.6), we characterize $\boldsymbol{w}^{\prime}$ and $\pi^{\prime}$ as a solution of

$$
\left\{\begin{aligned}
-\nu \Delta \boldsymbol{w}^{\prime}+{ }^{t} \nabla \boldsymbol{u} \boldsymbol{w}^{\prime}-\nabla \boldsymbol{w}^{\prime} \boldsymbol{u}+\nabla \pi^{\prime} & =\nabla \boldsymbol{w} \boldsymbol{u}^{\prime}-{ }^{t} \nabla \boldsymbol{u}^{\prime} \boldsymbol{w} & & \text { in } \Omega \backslash \bar{\omega} \\
\operatorname{div} \boldsymbol{w}^{\prime} & =0 & & \text { in } \Omega \backslash \bar{\omega} \\
\boldsymbol{w}^{\prime} & =2 m^{2}\left(\nu \partial_{\mathbf{n}} \boldsymbol{u}^{\prime}-p^{\prime} \mathbf{n}\right) & & \text { on } \partial \Omega \\
\boldsymbol{w}^{\prime} & =-\partial_{\mathbf{n}} \boldsymbol{w}(\boldsymbol{V} \cdot \mathbf{n}) & & \text { on } \partial \omega
\end{aligned}\right.
$$


with $\left\langle m^{2}\left(\nu \partial_{\mathbf{n}} \boldsymbol{w}^{\prime}-\pi^{\prime} \mathbf{n}\right), \mathbf{n}\right\rangle_{O}=0$. In particular, for $\omega=\omega^{*}, \nu \partial_{\mathbf{n}} \boldsymbol{u}-p \mathbf{n}=\boldsymbol{f}_{\boldsymbol{b}}$ on $O$. Therefore, according to the system (5.7) solved by $(\boldsymbol{w}, \pi)$, the uniqueness of the solution of the linearized Navier-Stokes problem enforces that $\boldsymbol{w}=\mathbf{0}$ in $\Omega \backslash \overline{\omega^{*}}$. Therefore $\partial_{\mathbf{n}} \boldsymbol{w}(\boldsymbol{V} \cdot \mathbf{n})=\mathbf{0}$ on $\partial \omega^{*}$ and we obtain the system 6.3 .

Third step: writing of $j^{\prime \prime}(0)$ as an integral on $\partial \omega$. We multiply the first equation of Problem 6.3 by $\boldsymbol{u}^{\prime}$ to get

$$
\int_{\Omega \backslash \overline{\omega^{*}}} \nu \nabla \boldsymbol{w}^{\prime}: \nabla \boldsymbol{u}^{\prime}+b_{\Omega \backslash \overline{\omega^{*}}}\left(\boldsymbol{u}^{\prime}, \boldsymbol{u}, \boldsymbol{w}^{\prime}\right)-b_{\Omega \backslash \overline{\omega^{*}}}\left(\boldsymbol{u}, \boldsymbol{w}^{\prime}, \boldsymbol{u}^{\prime}\right)=-\left\langle-\nu \partial_{\mathbf{n}} \boldsymbol{w}^{\prime}+\pi^{\prime} \mathbf{n}, \boldsymbol{u}^{\prime}\right\rangle_{\partial\left(\Omega \backslash \overline{\omega^{*}}\right)} .
$$

We multiply the first equation of Problem 5.5 by $\boldsymbol{w}^{\prime}$ to get

$$
\int_{\Omega \backslash \overline{\omega^{*}}} \nu \nabla \boldsymbol{u}^{\prime}: \nabla \boldsymbol{w}^{\prime}+b_{\Omega \backslash \overline{\omega^{*}}}\left(\boldsymbol{u}^{\prime}, \boldsymbol{u}, \boldsymbol{w}^{\prime}\right)+b_{\Omega \backslash \overline{\omega^{*}}}\left(\boldsymbol{u}, \boldsymbol{u}^{\prime}, \boldsymbol{w}^{\prime}\right)=-\left\langle-\nu \partial_{\mathbf{n}} \boldsymbol{u}^{\prime}+p^{\prime} \mathbf{n}, \boldsymbol{w}^{\prime}\right\rangle_{\partial\left(\Omega \backslash \overline{\omega^{*}}\right)} .
$$

Therefore, gathering $\sqrt{6.4}$ and $(6.5)$, and using $b_{\Omega \backslash \overline{\omega^{*}}}\left(\boldsymbol{u}, \boldsymbol{w}^{\prime}, \boldsymbol{u}^{\prime}\right)=-b_{\Omega \backslash \overline{\omega^{*}}}\left(\boldsymbol{u}, \boldsymbol{u}^{\prime}, \boldsymbol{w}\right)$, we obtain

$$
2 \int_{O} m^{2}\left|\nu \partial_{\mathbf{n}} \boldsymbol{u}^{\prime}-p^{\prime} \mathbf{n}\right|^{2}=-\int_{\partial \omega^{*}}\left(\nu \partial_{\mathbf{n}} \boldsymbol{w}^{\prime}-\pi^{\prime} \mathbf{n}\right) \cdot \partial_{\mathbf{n}} \boldsymbol{u}(\boldsymbol{V} \cdot \mathbf{n}) .
$$

\section{Justification of the instability}

We recall that we work now with more regularity concerning the object $\omega$ and the perturbation $\boldsymbol{V}$. The definitions of $\mathcal{O}_{\delta}, \boldsymbol{U}$ and $\mathcal{U}$ are now given by (6.1) and 6.2). Let us consider $\omega^{*} \in \mathcal{O}_{\delta}$ solution of the inverse problem $(3.3)$ in order to study the stability of the optimization problem (3.11) at $\omega^{*}$.

Proposition 7.1 (Compactness at a critical point). The Riesz operator corresponding to $\mathrm{D}^{2} J\left(\omega^{*}\right)$ defined from $\mathbf{H}^{1 / 2}\left(\partial \omega^{*}\right)$ to $\mathbf{H}^{-1 / 2}\left(\partial \omega^{*}\right)$ is compact.

This statement points out the lack of stability of the optimization problem (3.11). This compactness result means, roughly speaking, that, in a neighborhood of $\omega^{*}$ (i.e. for $t$ small), $J$ behaves as its second order approximation and one cannot expect an estimate of the kind $C t \leq \sqrt{J\left(\omega_{t}\right)}$ with a constant $C$ uniform in $\boldsymbol{V}$. Indeed, let us take the example of a starshaped domain $\omega$ in dimension two. Assume that $\partial \omega$ is parametrized by

$$
\begin{gathered}
\partial \omega=\left\{\left(\begin{array}{c}
g_{0} \\
g_{1}
\end{array}\right)+\right. \\
\left(g_{2}+\sum_{k=1}^{\infty}\left(g_{2 k+1} \cos (k \theta)+g_{2 k+2} \sin (k \theta)\right)\right)\left(\begin{array}{c}
\cos (\theta) \\
\sin (\theta)
\end{array}\right) \\
\left.=\sum_{k=0}^{\infty} g_{k} \boldsymbol{V}_{k}(\theta) ; \theta \in(0,2 \pi)\right\},
\end{gathered}
$$

where $g_{k} \in \mathbb{R}$. Then, since our identifiability result proves that the domain to be recovered is a local strict minimum of the least squares functional, we have, for all $n \in \mathbb{N}$, an estimate of the kind

$$
\forall \boldsymbol{V} \in \operatorname{Span}\left(\boldsymbol{V}_{k}\right)_{0 \leq k \leq 2 n+2}, \mathrm{D}^{2} J\left(\omega^{*}\right) \cdot(\boldsymbol{V}, \boldsymbol{V}) \geq C_{n}|\boldsymbol{V}|^{2},
$$

where $C_{n}$ is a positive constant. But this constant $C_{n}$ tends to 0 when $n$ tends to $+\infty$.

Hence, this proposition emphasizes that the gradient has not a uniform sensitivity with respect to the deformation directions: $J$ is degenerate for the high frequencies. This explains the numerical difficulties encountered to solve numerically this problem. For more details, we refer to [6, §2.3]. 
Justifying the ill-posedness of the problem. The compactness of the Riesz operator corresponding to the shape Hessian at possible solutions of the inverse problem is here proven using a local regularity argument as it is done in [6]. We do not use the hydrodynamical potential layers as it was done in [1] for the Laplacian case. This should provide an alternative proof.

We recall we assume that $\omega^{*} \in \mathcal{O}_{\delta}$ is a solution of the inverse problem $(3.3)$ with a $C^{2,1}$ boundary and that $\boldsymbol{V} \in \mathbf{W}^{3, \infty}\left(\mathbb{R}^{N}\right)$. To prove Proposition 7.1, we first decompose the shape Hessian at $\omega^{*}:$ for $\boldsymbol{V} \in \boldsymbol{U}$,

$$
\mathrm{D}^{2} J\left(\omega^{*}\right) \cdot \boldsymbol{V} \cdot \boldsymbol{V}=\left\langle M_{2} \circ M_{1}(\boldsymbol{V}), T_{u, p}(\boldsymbol{V})\right\rangle_{\partial \omega^{*}} .
$$

Here, $T_{u, p}: \mathbf{H}^{1 / 2}\left(\partial \omega^{*}\right) \rightarrow \mathbf{H}^{1 / 2}\left(\partial \omega^{*}\right)$ is defined by

$$
T_{u, p}(\boldsymbol{V}):=-\partial_{\mathbf{n}} \boldsymbol{u}(\boldsymbol{V} \cdot \mathbf{n}),
$$

and $M_{1}: \mathbf{H}^{1 / 2}\left(\partial \omega^{*}\right) \rightarrow \mathbf{H}^{1 / 2}(\partial \Omega)$ by $M_{1}(\boldsymbol{V}):=2 m^{2}\left(\nu \partial_{\mathbf{n}} \boldsymbol{u}^{\prime}-p^{\prime} \mathbf{n}\right)$, with $\left(\boldsymbol{u}^{\prime}, p^{\prime}\right)$ the solution of 5.5). Finally, $M_{2}: \mathbf{H}^{1 / 2}(\partial \Omega) \rightarrow \mathbf{H}^{-1 / 2}\left(\partial \omega^{*}\right)$ satisfies $M_{2}(\boldsymbol{\varphi}):=\nu \partial_{\mathbf{n}} \boldsymbol{\psi}-\chi \mathbf{n}$, where $(\boldsymbol{\psi}, \chi)$ is the solution of the following problem

$$
\left\{\begin{array}{rlll}
-\nu \Delta \psi+{ }^{t} \nabla \boldsymbol{u} \boldsymbol{\psi}-\nabla \boldsymbol{\psi} \boldsymbol{u}+\nabla \chi & =\mathbf{0} & & \text { in } \Omega \backslash \overline{\omega^{*}} \\
\operatorname{div} \boldsymbol{\psi} & =0 & & \text { in } \Omega \backslash \overline{\omega^{*}} \\
\boldsymbol{\psi} & = & \boldsymbol{\varphi} & \text { on } \partial \Omega \\
\boldsymbol{\psi} & =\mathbf{0} & & \text { on } \partial \omega^{*} .
\end{array}\right.
$$

We study each operator: Lemma 7.2 states that $T_{u, p}$ and $M_{1}$ are linear continuous and Lemma 7.3 claims that $M_{2}$ is compact. Hence, compactness is obtained by composition of linear continuous operator with a compact one.

Lemma 7.2. The operators $T_{u, p}$ and $M_{1}$ are linear continuous.

Proof. Since $\boldsymbol{u}$ does not depend on $\boldsymbol{V}$ and using the continuity of the gradient operator $\nabla$, the operator $T_{u, p}$ is linear continuous as multiplier by a smooth function (see [22]).

Let us consider $\boldsymbol{V} \in \mathbf{H}^{1 / 2}\left(\partial \omega^{*}\right)$. Using a local regularity result similar to Theorem 2.8 , we prove that $(\boldsymbol{v}, q)$ belongs to $\mathbf{H}^{2} \times \mathrm{H}^{1}$ in a neighborhood of $O$ (since $\widetilde{O}$ is $C^{1,1}$ ) and, since $\operatorname{supp}(m)=\bar{O}$, the operator

$$
\Phi: \boldsymbol{B} \in \mathbf{H}^{1 / 2}\left(\partial \omega^{*}\right) \mapsto 2 m^{2}\left(\nu \partial_{\mathbf{n}} \boldsymbol{v}-q \mathbf{n}\right) \in \mathbf{H}^{1 / 2}(\partial \Omega),
$$

where $(\boldsymbol{v}, q)$ is the solution of the problem

$$
\left\{\begin{aligned}
-\nu \Delta \boldsymbol{v}+(\boldsymbol{v} \cdot \nabla) \boldsymbol{u}+(\boldsymbol{u} \cdot \nabla) \boldsymbol{v}+\nabla q & =\mathbf{0} & & \text { in } \Omega \backslash \overline{\omega^{*}} \\
\operatorname{div} \boldsymbol{v} & =0 & & \text { in } \Omega \backslash \overline{\omega^{*}} \\
\boldsymbol{v} & =\mathbf{0} & & \text { on } \partial \Omega \\
\boldsymbol{v} & =\boldsymbol{B} & & \text { on } \partial \omega^{*}
\end{aligned}\right.
$$

with $\int_{O} m^{2}\left(\nu \partial_{\mathbf{n}} \boldsymbol{v}-q \mathbf{n}\right) \cdot \mathbf{n}=0$, is linear continuous. Finally, by composition, $M_{1}=\Phi \circ T_{u, p}$ is linear continuous from $\mathbf{H}^{1 / 2}\left(\partial \omega^{*}\right)$ into $\mathbf{H}^{1 / 2}(\partial \Omega)$.

Lemma 7.3. The operator $M_{2}: \mathbf{H}^{1 / 2}(\partial \Omega) \rightarrow \mathbf{H}^{-1 / 2}\left(\partial \omega^{*}\right)$ is compact. 
Proof. According to a local regularity result similar to Theorem 2.8, the operator

$$
\varphi_{1}: \varphi \in \mathbf{H}^{1 / 2}(\partial \Omega) \mapsto(\boldsymbol{\psi}, \chi) \in \mathbf{H}^{3}\left(\Omega_{\delta} \backslash \overline{\omega^{*}}\right) \times \mathrm{H}^{2}\left(\Omega_{\delta} \backslash \overline{\omega^{*}}\right),
$$

where $(\boldsymbol{\psi}, \chi)$ is the solution of Problem (7.1), is linear continuous. Moreover,

$$
\varphi_{2}:(\boldsymbol{\psi}, \chi) \in \mathbf{H}^{3}\left(\Omega_{\delta} \backslash \overline{\omega^{*}}\right) \times \mathrm{H}^{2}\left(\Omega_{\delta} \backslash \overline{\omega^{*}}\right) \mapsto \nu \partial_{\mathbf{n}} \boldsymbol{\psi}-\chi \mathbf{n} \in \mathbf{H}^{3 / 2}\left(\partial \omega^{*}\right)
$$

is linear continuous and the embedding of $\mathbf{H}^{3 / 2}\left(\partial \omega^{*}\right)$ in $\mathbf{H}^{-1 / 2}\left(\partial \omega^{*}\right)$, denoted by $\varphi_{3}$, is compact. Thus, we get, by composition, that the operator $M_{2}=\varphi_{3} \circ \varphi_{2} \circ \varphi_{1}$ is compact.

Remark 7.4. We see in this proof that if $\nu \partial_{\mathbf{n}} \psi-\chi \mathbf{n} \in \mathbf{H}^{k+1 / 2}\left(\partial \omega^{*}\right)$, for a $k \in \mathbb{N}^{*}$, we use the compactness of the embedding of $\mathbf{H}^{k+1 / 2}\left(\partial \omega^{*}\right)$ in $\mathbf{H}^{-1 / 2}\left(\partial \omega^{*}\right)$. Thus, the more $\omega^{*}$ is smooth (with a $C^{k+1,1}$ boundary), the more $\nu \partial_{\mathbf{n}} \boldsymbol{\psi}-\chi \mathbf{n}$ is regular (it belongs to $\mathbf{H}^{k+1 / 2}\left(\partial \omega^{*}\right)$ ), the more the functional $J$ is degenerate (see the comment after the statement of Proposition 7.1) and the more the object is difficult to detect. For example, a circle (for $N=2$ ) is very difficult to detect, if we do not know that we are looking for a circle.

Acknowledgments This work is part of the project ANR-09-BLAN-0037 Geometric analysis of optimal shapes (GAOS) financed by the French Agence Nationale de la Recherche (ANR). I also thank Marc Dambrine and Mehdi Badra to suggest me to study this problem and for many helpful discussions.

\section{References}

[1] L. Afraites, M. Dambrine, K. Eppler, and D. Kateb. Detecting perfectly insulated obstacles by shape optimization techniques of order two. Discrete Contin. Dyn. Syst. Ser. B, 8(2):389-416 (electronic), 2007.

[2] L. Afraites, M. Dambrine, and D. Kateb. On second order shape optimization methods for electrical impedance tomography. SIAM J. Control Optim., 47(3):1556-1590, 2008.

[3] C. Alvarez, C. Conca, L. Friz, O. Kavian, and J. H. Ortega. Identification of immersed obstacles via boundary measurements. Inverse Problems, 21(5):1531-1552, 2005.

[4] C. Amrouche and V. Girault. Decomposition of vector spaces and application to the Stokes problem in arbitrary dimension. Czechoslovak Math. J., 44(119)(1):109-140, 1994.

[5] C. Amrouche and M. Á. Rodríguez-Bellido. Stationary Stokes, Oseen and NavierStokes equations with singular data. Arch. Ration. Mech. Anal., 199(2):597-651, 2011.

[6] M. Badra, F. Caubet, and M. Dambrine. Detecting an obstacle immersed in a fluid by shape optimization methods. M3AS, 21(10):2069-2101, 2011.

[7] J. A. Bello, E. Fernández Cara, and J. Simon. Optimal shape design for NavierStokes flow. In System modelling and optimization (Zurich, 1991), volume 180 of Lecture Notes in Control and Inform. Sci., pages 481-489. Springer, Berlin, 1992.

[8] J. A. Bello, E. Fernández Cara, and J. Simon. The variation of the drag with respect to the domain in Navier-Stokes flow. In Optimization, optimal control and partial differential equations (Iaşi, 1992), volume 107 of Internat. Ser. Numer. Math., pages 287-296. Birkhäuser, Basel, 1992. 
[9] J. Bergh and J. Löfström. Interpolation spaces. An introduction. Springer-Verlag, Berlin, 1976. Grundlehren der Mathematischen Wissenschaften, No. 223.

[10] C. Conca, P. Cumsille, J. Ortega, and L. Rosier. Detecting a moving obstacle in an ideal fluid by a boundary measurement. C. R. Math. Acad. Sci. Paris, 346(15-16):839$844,2008$.

[11] C. Conca, M. Malik, and A. Munnier. Detection of a moving rigid solid in a perfect fluid. Inverse Problems, 26(9):095010, 18, 2010.

[12] P. Constantin and C. Foias. Navier-Stokes equations. Chicago Lectures in Mathematics. University of Chicago Press, Chicago, IL, 1988.

[13] A. Doubova, E. Fernández-Cara, and J. H. Ortega. On the identification of a single body immersed in a Navier-Stokes fluid. European J. Appl. Math., 18(1):57-80, 2007.

[14] K. Eppler and H. Harbrecht. A regularized Newton method in electrical impedance tomography using shape Hessian information. Control Cybernet., 34(1):203-225, 2005.

[15] C. Fabre and G. Lebeau. Prolongement unique des solutions de l'equation de Stokes. Comm. Partial Differential Equations, 21(3-4):573-596, 1996.

[16] G. P. Galdi. An introduction to the mathematical theory of the Navier-Stokes equations., volume 38 of Springer Tracts in Natural Philosophy. Springer-Verlag, New York, 1994. Linearized steady problems.

[17] V. Girault and P.-A. Raviart. Finite element methods for Navier-Stokes equations, volume 5 of Springer Series in Computational Mathematics. Springer-Verlag, Berlin, 1986. Theory and algorithms.

[18] P. Grisvard. Elliptic problems in nonsmooth domains, volume 24 of Monographs and Studies in Mathematics. Pitman (Advanced Publishing Program), Boston, MA, 1985.

[19] A. Henrot and M. Pierre. Variation et optimisation de formes, volume 48 of Mathématiques 8 Applications (Berlin) [Mathematics \& Applications]. Springer, Berlin, 2005. Une analyse géométrique. [A geometric analysis].

[20] A. Henrot and Y. Privat. Une conduite cylindrique n'est pas optimale pour minimiser l'énergie dissipée par un fluide. C. R. Math. Acad. Sci. Paris, 346(19-20):1057-1061, 2008.

[21] A. Henrot and Y. Privat. What is the optimal shape of a pipe? Arch. Ration. Mech. Anal., 196(1):281-302, 2010.

[22] V. Maz'ya and T. Shaposhnikova. Theory of multipliers in spaces of differentiable functions, volume 23 of Monographs and Studies in Mathematics. Pitman (Advanced Publishing Program), Boston, MA, 1985.

[23] F. Murat and J. Simon. Sur le contrôle par un domaine géométrique. Rapport du L.A. 189, 1976. Université de Paris VI, France.

[24] J. Simon. Domain variation for drag in Stokes flow. In Control theory of distributed parameter systems and applications (Shanghai, 1990), volume 159 of Lecture Notes in Control and Inform. Sci., pages 28-42. Springer, Berlin, 1991. 
[25] R. Temam. Navier-Stokes equations. AMS Chelsea Publishing, Providence, RI, 2001. Theory and numerical analysis, Reprint of the 1984 edition. 